Florida International University FIU Digital Commons

$1-17-2013$

\title{
An Investigation of Vertical Turbulent Transport Processes in Coastal Regions Using Tower Observations
}

Jonathan Joseph Furst

Florida International University, jfurs001@fiu.edu

DOI: $10.25148 /$ etd.FI13040303

Follow this and additional works at: https://digitalcommons.fiu.edu/etd

\section{Recommended Citation}

Furst, Jonathan Joseph, "An Investigation of Vertical Turbulent Transport Processes in Coastal Regions Using Tower Observations" (2013). FIU Electronic Theses and Dissertations. 814.

https://digitalcommons.fiu.edu/etd/814 


\title{
FLORIDA INTERNATIONAL UNIVERSITY
}

Miami, Florida

\section{AN INVESTIGATION OF VERTICAL TURBULENT TRANSPORT PROCESSES IN COASTAL REGIONS USING TOWER OBSERVATIONS}

\author{
A thesis submitted in partial fulfillment of \\ the requirements for the degree of \\ MASTER OF SCIENCE \\ in \\ GEOSCIENCES \\ by
}

Jonathan Furst 
To: Dean Kenneth G. Furton

College of Arts and Sciences

This thesis, written by Jonathan Furst, and entitled An Investigation of Vertical Turbulent Transport Processes in Coastal Regions Using Tower Observations, having been approved in respect to style and intellectual content, is referred to you for judgment.

We have read this thesis and recommend that it be approved.

$\begin{array}{r}\hline \text { Haiyan Jiang } \\ \hline \text { Hugh Willoughby } \\ \hline \text { Ping Zhu, Major Professor }\end{array}$

Date of Defense: January 17, 2013

The thesis of Jonathan Furst is approved.

$\begin{array}{r}\begin{array}{r}\text { Dean Kenneth G. Furton } \\ \text { College of Arts and Sciences }\end{array} \\ \hline \begin{array}{r}\text { Dean Lakshmi N. Reddi } \\ \text { University Graduate School }\end{array}\end{array}$

Florida International University, 2013 


\section{ACKNOWLEDGMENTS}

I would like to thank my advisor Dr. Ping Zhu for providing motivation and support throughout my time at FIU. My thesis research would not have been possible without him. I would also like to thank Dr. Hugh Willoughby for his advice and guidance, both academic and personal. Thank you to Dr. Haiyan Jiang for serving on my thesis committee. In addition to my thesis committee, I also express gratitude to several fellow students and colleagues who provided me assistance in times of need: Joseph Zagrodnik, Zhenduo Zhu, Cen Gao, Walter Conklin, Jimmy Erwin and Roy LiuMarques. My family has been there for me throughout my graduate career and I owe my overall success to their unconditional support. 


\begin{abstract}
OF THE THESIS
AN INVESTIGATION OF VERTICAL TURBULENT TRANSPORT PROCESSES IN COASTAL REGIONS USING TOWER OBSERVATIONS

by

Jonathan Furst

Florida International University, 2013

Miami, Florida

Professor Ping Zhu, Major Professor

High-resolution tower observations of turbulent transport processes in the coastal atmospheric surface layer show that the exchange coefficients for momentum, enthalpy, and moisture behave differently for different environmental and atmospheric conditions. The drag coefficient is closely tied to wind speed and turbulent intensity. The exchange coefficient for enthalpy shows a dependence on stability. Analysis of the turbulent kinetic energy budget yields a new parameterization framework that well explains the observed variation of the drag coefficient, particularly at low wind speeds.
\end{abstract}




\section{TABLE OF CONTENTS}

CHAPTER

PAGE

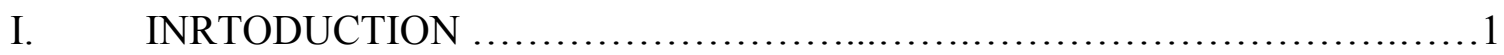

1.1 Turbulence and the Atmospheric Surface Layer...............................

1.2 The Bulk Transfer Parameterization of Surface Turbulent Fluxes ..............3

1.3 Scientific Questions...................................................... 8

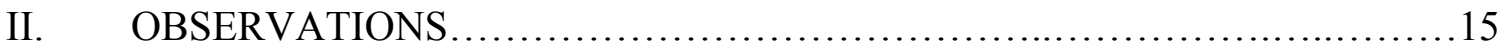

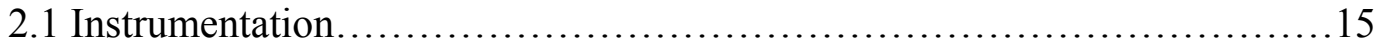

2.1.1 IHRC 10-m PWT ............................................

2.1.2 Eddy Covariance Observing System............................16

2.2 Tower Deployments..............................................21

2.2.1 Everglades National Park - Lake Chekika Deployment..............21

2.2.2 Biscayne Bay Deployment....................................23

2.2.3 Naples Municipal Airport - Tropical Storm Isaac..................24

2.2.4 Waveland, MS - Tropical Storm/Hurricane Isaac.................25

III. DATA ANALYSIS AND METHODS .....................................28

3.1 Eddy Correlation Method.............................................28

3.2 Quality Control and Assurance Procedures...............................29

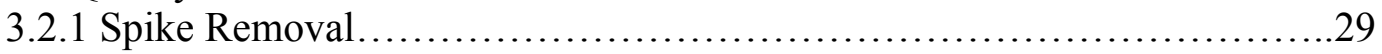

3.2.2 Sonic Anemometer Tilt Correction......................................30

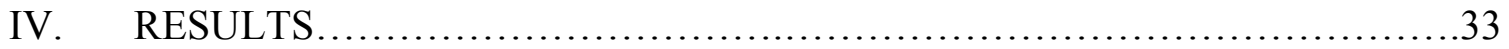

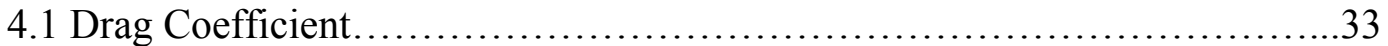

4.2 A New Perspective on Bulk Exchange Coefficients.........................4 41

4.2.1 Neutral Condition.............................................42

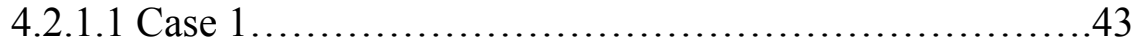

4.2.1.2 Case 2.............................................. 44

4.2.2 Non Neutral Condition........................................49

4.3 Exchange Coefficients for Heat and Moisture................................53

4.3.1 Tropical Cyclones and Enthalpy Flux..............................61

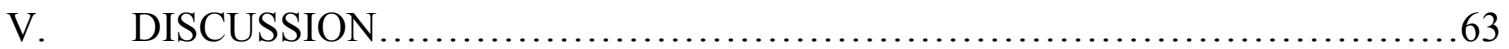

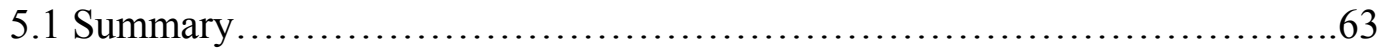

5.2 Results and Conclusions...................................................64

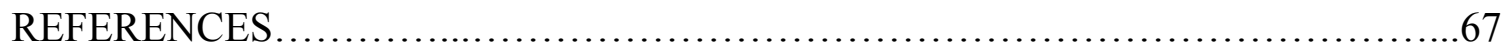

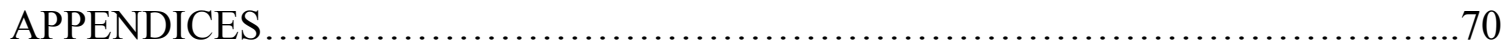




\section{LIST OF FIGURES}

FIGURE

PAGE

1.1 Schematic illustrating the effect of a passing eddy on a pair of air particles.........3

1.2 Plot adopted from Black et al. of observed $C_{D N}$ against 10-m wind speeds. Also plotted is the theoretical line of $C_{D N}$ (thick blue)........................... 10

1.3 Plot of $C_{D N}$ against wind speed obtained from multiple field experiments over

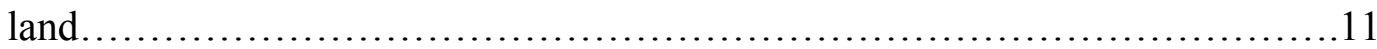

1.4 Plot of the computed aerodynamic surface roughness against wind speed that is required to obtain a given $C_{D N}$ value represented by the best fit curve in Figure 1.3 13

2.1 Photo of the PWT set up behind the FIU Engineering Campus..................16

2.2 Photo of the sonic anemometer (right) and gas analyzer (left) atop the PWT. Also shown are the pre-existing propeller anemometers (bottom) and wind vane monitor (top) ................................................... 17

2.3 Photo of the ground temperature sensors................................ 21

2.4 Aerial view of the PWT deployment location at the ENP site. The small yellow and black target symbol is the location of the tower when it was deployed........22

2.5 Aerial view of the PWT deployment location at BBC. The blue outline is BBC and the small yellow and black target symbol is the location of the tower when it was deployed.

2.6 Satellite image of Hurricane Sandy passing by the Florida peninsula. The red dot indicates the location of the tower at $\mathrm{BBC}$. .24

2.7 Aerial view of the PWT deployment location at the NMA site. The red line in the upper map is an approximation to the track of Tropical Storm Isaac............25

2.8 Aerial view of the PWT deployment location at the Waveland site. The red line in the upper map is an approximation to the track of Tropical Storm/Hurricane Isaac 
4.1 Plots of friction velocity, kinematic sensible heat flux, kinematic moisture flux, and TKE comparing the two measurement heights (5-m and 10-m) at the ENP site .34

4.2 Plot of estimated values of $C_{D}$ against $10-\mathrm{m}$ wind speed for all four sites. .35

4.3 Plot of $C_{D}$ vs. the stability parameter $\varsigma=\frac{z}{L}$ for all four sites

4.4 Comparison of $C_{D}$ vs. $\varsigma=\frac{z}{L}$ for two roughness lengths as predicted by MOS..37

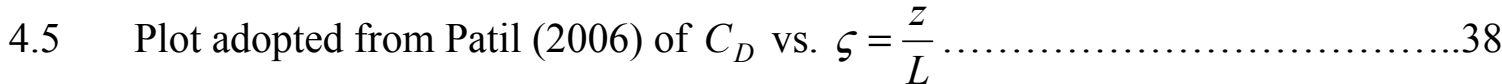

4.6 Plot of $C_{D}$ against TKE for all data collected at all four sites.................39

4.7 Logarithmic plot of the ratio of $C_{D}$ to TKE against 10-m wind speed for all four sites as well as data collected in Hurricane Ivan (2004) 40

4.8 Logarithmic plot of the total momentum flux against TKE for all four sites, including Hurricane Ivan (2004) 41

4.9 Plot of the energy density power spectra of $u, v$, and $w$ for an arbitrary 15 minute spectral lag from the ENP data. 46

4.10 Plot of the estimated energy dissipation rate $\left(\varepsilon_{e}\right)$ at each frequency in the inertial sub-range

4.11 Plot of the estimated coefficient $\kappa=c_{1}^{3 / 2} c_{2}$ against $10-\mathrm{m}$ wind speed for all data from all four sites including Hurricane Ivan (2004). .48

4.12 Plot of $C_{D N}$ values computed from MOS (blue) and from equation 4.15 (red) against 10-m wind speed. .49

4.13 Plot of $C_{D}$ as a function of both $10-\mathrm{m}$ wind speed and the stability parameter $\varsigma=\frac{z}{L}$

4.14 Plot of $C_{D}$ against $\varsigma=\frac{z}{L}$ for different wind speed ranges..................52 
4.15 Time series of wind speed, buoyancy flux, and exchange coefficients for the ENP

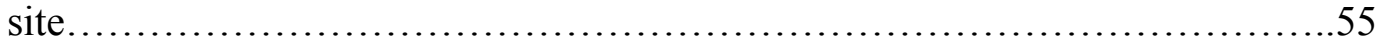

4.16 Time series of wind speed, buoyancy flux, and exchange coefficients for the BBC

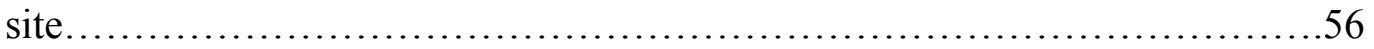

4.17 Plot of $C_{H}$ (left panel) and $C_{Q}$ (right panel) against 10-m wind speed for both the

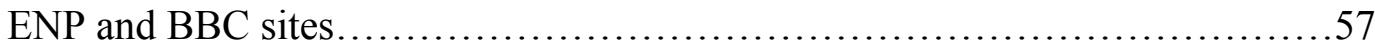

4.18 Plot of $C_{H}$ (left panel) and $C_{Q}$ (right panel) against $\varsigma=\frac{z}{L}$ for both the ENP and $\mathrm{BBC}$

sites

4.19 Plot (on a logarithmic coordinate) of the ratio of $C_{H}$ to TKE (left panel) and $C_{Q}$ to TKE (right panel) against 10-m wind speed for both the ENP and BBC sites..60

4.20 Plot of the ratio of enthalpy flux to $C_{D}$ (left panel) and ratio of $C_{Q}$ to $C_{H}$ (right panel) against 10-m wind speed for both the ENP and BBC sites...............62 


\section{LIST OF ABBREVIATIONS, ACRONYMS, AND SYMBOLS}

\section{BBC Biscayne Bay Campus}

$C_{D} \quad$ Bulk transfer coefficient for momentum, drag coefficient (dimensionless)

$C_{D N} \quad$ Neutral drag coefficient

$C_{H} \quad$ Bulk transfer coefficient for heat (dimensionless)

$C_{Q} \quad$ Bulk transfer coefficient for moisture (dimensionless)

DR Double rotation method

ENP Everglades National Park

IHRC International Hurricane Research Center (FIU)

$L \quad$ Obukhov Length (m)

LHS Left hand side

MOS Monin Obukhov Similarity (theory)

$P \quad$ Pressure $(\mathrm{hPa})$

PWT Portable Wind Tower

RHS Right hand side

$r \quad$ Water vapor mixing ratio $(\mathrm{g} / \mathrm{kg})$

SL Surface Layer

SGS Sub-grid scale

$T \quad$ Temperature $\left({ }^{\circ} \mathrm{C}\right)$

TKE Turbulent Kinetic Energy

$T_{S} \quad$ Sonic temperature $\left({ }^{\circ} \mathrm{C}\right)$

$T_{v} \quad$ Virtual temperature $\left({ }^{\circ} \mathrm{C}\right)$

$t \quad$ Time (unit varies)

$U \quad$ Total wind $\left(\mathrm{ms}^{-1}\right)$ 


$\begin{array}{ll}u_{*} & \text { Friction velocity }\left(\mathrm{ms}^{-1}\right) \\ u & \text { Along wind component }\left(\mathrm{ms}^{-1}\right) \\ v & \text { Cross wind component }\left(\mathrm{ms}^{-1}\right) \\ w & \text { Vertical wind component }\left(\mathrm{ms}^{-1}\right) \\ \text { WMP } & \text { WindMaster Pro (Sonic Anemometer) } \\ z & \quad \text { Height above ground measurements are observed }(10 \mathrm{~m}) \\ z_{0} & \text { Aerodynamic roughness length (m) } \\ \theta & \text { Potential temperature }\left({ }^{\circ} \mathrm{C}\right) \\ \varsigma & \text { Surface layer stability parameter (dimensionless) } \\ \kappa & \text { Von-Karman constant } \\ \varepsilon_{\mathrm{e}} & \text { TKE dissipation rate } \\ \Lambda & \text { Empirical dissipation length scale }\end{array}$




\section{INTRODUCTION}

\subsection{Turbulence and the Atmospheric Surface Layer}

The atmospheric surface layer (SL) is defined as the lowest $10 \%$ of the atmospheric boundary layer. Processes in the SL, which are directly influenced by the presence of the earth's surface, are dominated by atmospheric motions with time scales typically less than one hour. These high frequency atmospheric perturbations (with respect to the mean flow) are also known as turbulence. Although large turbulent eddies can possess well defined structures and organize into coherent circulations (Christen et al. 2007), turbulent motions in nature are chaotic. This chaotic nature makes it impossible to represent turbulent eddies individually, and therefore, in practice, turbulent motions in the SL can only be treated statistically.

The importance of SL turbulence lies in the interaction between the Earth's surface and the overlying atmosphere. Atmospheric turbulence is generated from frictional drag and/or heterogeneous surface heating. The turbulent motions provide a mechanism to promote the exchange of energy, moisture, and momentum between the Earth's surface and atmosphere. The vertical fluxes of heat and moisture carried by SL turbulence are the driving forces for atmospheric motions. Similarly, oceanic surface currents are driven by the surface wind stress. It is through the surface turbulent fluxes that the atmosphere-ocean-land system is coupled.

The vertical transport generated by turbulence is schematically illustrated in Figure 1.1, where the surface sensible heat flux is considered as an example. The thick solid curve in Figure 1.1 represents the mean vertical profile of potential temperature in the SL. Consider the two points $\mathrm{A}$ and $\mathrm{B}$ in the profile, which are subjected to an 
arbitrary turbulent eddy. The eddy perturbs the atmosphere so that the air particle at A experiences an initial downward motion (i.e., w’<0). As soon as the particle moves downward, it "feels" colder than the environment under an assumed stratification, where potential temperature decreases with height (i.e., $\theta^{\prime}<0$ ), thereby yielding a positive sensible heat flux, w' $\theta^{\prime}>0$. In the meantime, the air particle at B subjected to the same eddy experiences an initial upward motion (i.e., w'>0) and a warm bias (i.e., $\theta^{\prime}>0$ ) as it moves to a new environment, which also yields a positive sensible heat flux, w' $\theta^{\prime}>0$. The sign of $w^{\prime} \theta$ ' is positive for both particles despite the fact that the atmospheric perturbations generated by the eddy at the two points are off-phase. The relationship between w' and $\theta$ ' holds regardless of the size and orientation of eddies, and thus, the net result is to generate a positive heat flux under the assumed stratification. Similarly, a net negative heat flux is generated in a stably stratified atmosphere (i.e., when the mean potential temperature increases with height).

The same argument can be applied to the vertical transport of moisture and momentum as well. This example indicates that the vertical turbulent fluxes in the SL can be mathematically expressed as the covariance of the perturbations of vertical velocity and a generic scalar. Although the concept of vertical transport induced by SL turbulence can be readily understood, a quantitative theory that effectively explains fluxes of heat, moisture, and momentum is more difficult. An accurate estimate of covariance requires detailed information of the chaotic turbulent eddies that span over a spectrum of time and length scales. Unfortunately, this information can be obtained only from high resolution meteorological instruments that are capable of recording high frequency turbulent 
perturbations. A central problem in the field of boundary layer meteorology is appropriate determination of surface turbulent fluxes from conventional meteorological measurements or from the mean state of the atmosphere. Although this has been widely studied, it continues to be a challenge for observational meteorology and numerical simulations.

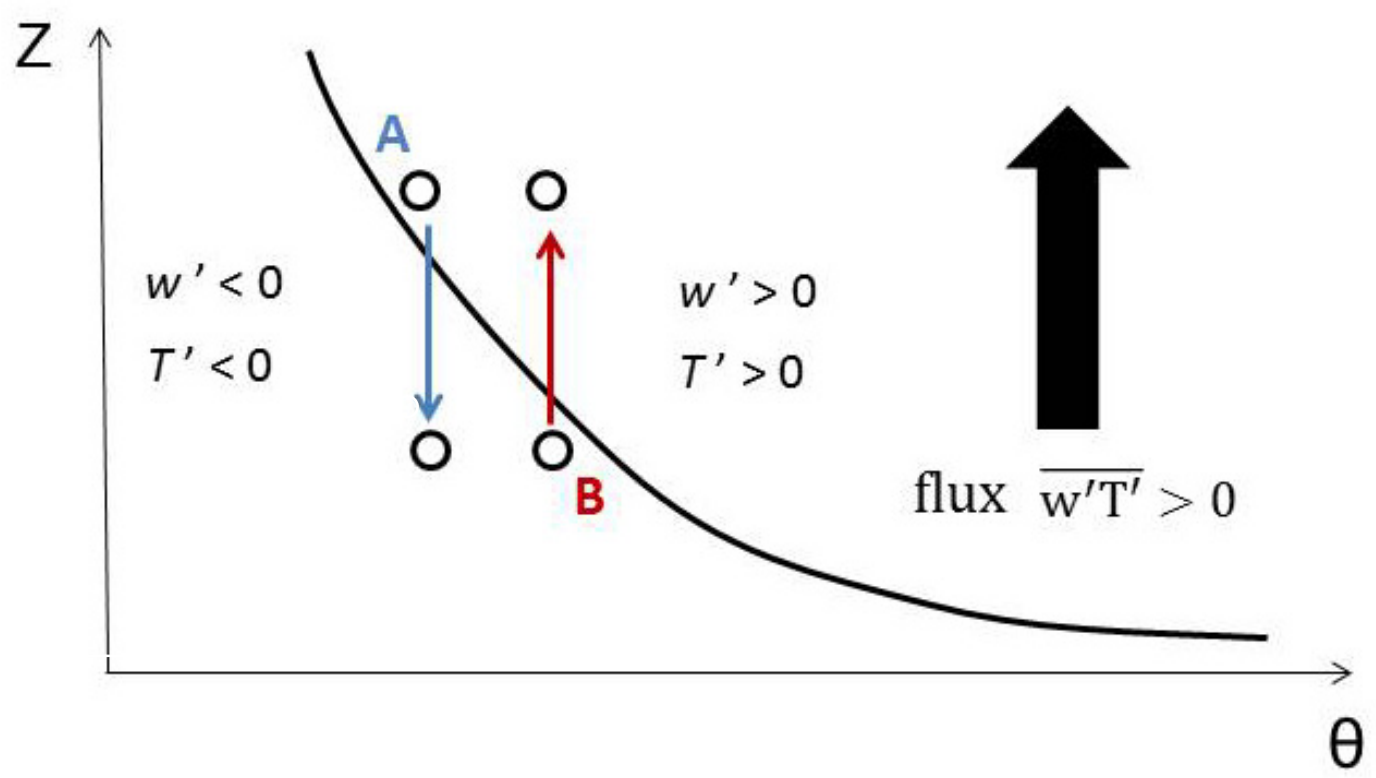

Figure 1.1 Schematic illustrating the effect of a passing eddy on a pair of air particles.

\subsection{The Bulk Transfer Parameterization of Surface Turbulent Fluxes}

As stated previously, the atmospheric perturbations induced by turbulent eddies cannot be obtained from conventional observations and are considered sub-grid scale (SGS) processes in numerical simulations. Thus, the surface turbulent fluxes have to be parameterized from the mean state of the atmosphere or from model resolved variables when high resolution data is unavailable. Currently, in observational analyses and 
numerical simulations, with almost no exception, the surface fluxes are determined through the bulk transfer parameterization method represented as follows:

$$
\begin{array}{r}
u_{*}^{2}=C_{D} \bar{U}^{2}, \quad(1.1) \\
\left(\overline{w^{\prime} \theta^{\prime}}\right)_{s}=-C_{H} \bar{U}\left(\bar{\theta}-\theta_{G}\right), \\
\left(\overline{w^{\prime} q^{\prime}}\right)_{s}=-C_{Q} \bar{U}\left(\bar{q}-q_{G}\right),
\end{array}
$$

where $u_{*}$ is the frictional velocity defined as $u_{*}=\left({\overline{u^{\prime} w^{\prime}}}^{2}+{\overline{v^{\prime} w^{\prime}}}^{2}\right)^{1 / 4}, \quad \overline{u^{\prime} w^{\prime}}, \overline{v^{\prime} w^{\prime}}$, $\left(\overline{w^{\prime} \theta^{\prime}}\right)_{s}$, and $\left(\overline{w^{\prime} q^{\prime}}\right)_{s}$ are the surface kinematic momentum, sensible heat, and moisture fluxes, respectively. $\bar{U}, \bar{\theta}$, and $\bar{q}$ represent the wind speed, atmospheric potential temperature, and water vapor mixing ratio at the reference height, typically $10 \mathrm{~m}$ above the surface; $\theta_{G}$ and $q_{G}$ are the potential temperature and water vapor mixing ratio "on the ground surface", which is typically a few centimeters above the ground surface (Stull 1988). $C_{D}, C_{H}$ and $C_{Q}$ are the dimensionless exchange coefficients for momentum, heat, and moisture, and are often called the drag coefficient, Stanton number, and Dalton number respectively in the literature. Previous studies (e.g., Mahrt et al. 2001, Garratt 1977, Grachev et al. 2001) show that the exchange coefficients are not constant but are instead functions of wind speed and atmospheric stability. Thus, the key to the success of this approach is to accurately determine the aforementioned exchanges.

In practice, to close the system described by equations $1.1-1.3$, the bulk transfer parameterization is often combined with the Monin-Obukhov similarity theory (hereafter 
referred to as MOS). From dimensional analysis, or Buckingham's (19XX) $\pi$ theorem, Monin and Obukhov (1954) first showed that the vertical gradient of SL mean properties can be related to turbulence fluxes through:

$$
\begin{aligned}
& \frac{\kappa z}{u_{*}} \frac{\partial \bar{u}}{\partial z}=\phi_{m}(\varsigma), \\
& \frac{\kappa z}{\theta_{*}} \frac{\partial \bar{\theta}}{\partial z}=\phi_{h}(\varsigma), \\
& \frac{\kappa z}{q_{*}} \frac{\partial \bar{q}}{\partial z}=\phi_{q}(\varsigma),
\end{aligned}
$$

where, $\kappa$ is the Von-Karman constant. $\bar{u}, \bar{\theta}$ and $\bar{q}$ represent the mean wind speed, potential temperature, and water vapor mixing ratio in the SL, respectively; $\theta_{*}$ and $q_{*}$ are the temperature scale and moisture scale defined as $\theta_{*}=\frac{\overline{w^{\prime} \theta^{\prime}}}{u_{*}}$ and $q_{*}=\frac{\overline{w^{\prime} q^{\prime}}}{u_{*}} \cdot \varsigma=\frac{z}{L}$ is the dimensionless stability parameter, where $z$ is the height and $L$ is the Monin-Obukhov length defined as $L=\frac{-u_{*}^{3} \bar{\theta}}{k g \overline{w^{\prime} \theta^{\prime}}}$. Expressions (1.4) - (1.6) are known as the empirical dimensionless functions of atmospheric stability, $\phi_{m}, \phi_{h}$ and $\phi_{q}$. The empirical functions cannot be derived from $\pi$ theorem directly, but instead must be determined from laboratory and/or field experiments. The most recognized and widely accepted empirical dimensionless functions are those obtained by Businger et al. (1971). On the basis of their famous Kansas experiment, the dimensionless empirical functions for $\phi_{m}$ and $\phi_{h}$ are as follows: 


$$
\begin{aligned}
& \left\{\begin{array}{lll}
\phi_{m}=(1-16 \varsigma)^{-1 / 4} & \text { for } & \varsigma<0 \\
\phi_{m}=1+5 \varsigma & \text { for } & \varsigma>0
\end{array},\right.
\end{aligned}
$$

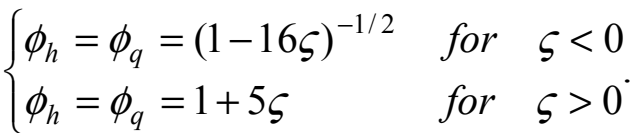

Utilizing equations $1.7-1.8$, the MOS relationships can be integrated to yield:

$$
\begin{gathered}
\bar{u}=\frac{u_{*}}{\kappa}\left[\ln \left(\frac{z}{z_{0}}\right)-\Psi_{m}(\varsigma)\right], \\
\bar{\theta}-\theta_{G}=\frac{\theta_{*}}{\kappa}\left[\ln \left(\frac{z}{z_{0 \theta}}\right)-\Psi_{h}(\varsigma)\right], \\
\bar{q}-q_{G}=\frac{q_{*}}{\kappa}\left[\ln \left(\frac{z}{z_{0 q}}\right)-\Psi_{q}(\varsigma)\right],
\end{gathered}
$$

where:

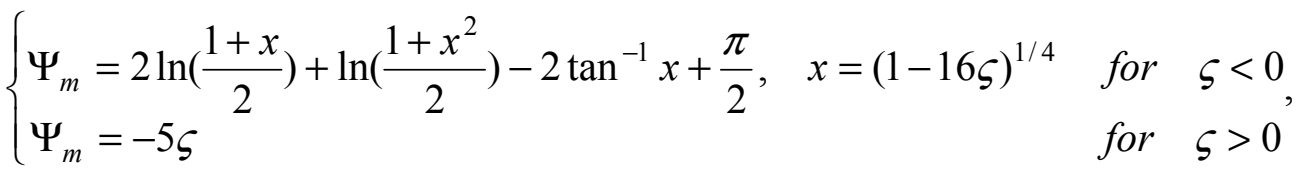

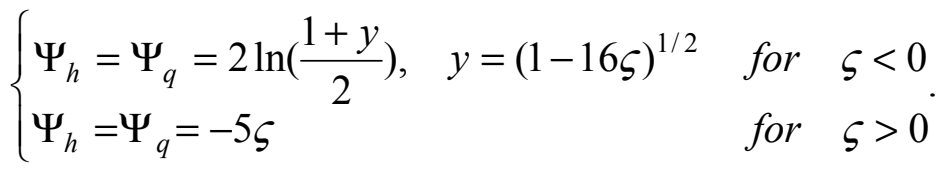

$z_{0}, z_{0 \theta}$ and $z_{0 q}$ are the aerodynamic surface roughness for wind, potential temperature, and water vapor.

Combining equations $1.1-1.3$ with equations $1.9-1.11$, it is easy to show: 


$$
\begin{gathered}
C_{D}=\frac{\kappa^{2}}{\left[\ln \left(\frac{z}{z_{0}}\right)-\Psi_{m}(\varsigma)\right]^{2}}, \quad C_{D N}=\frac{\kappa^{2}}{\left[\ln \left(\frac{z}{z_{0}}\right)\right]^{2}}, \\
C_{H}=\frac{\kappa^{2}}{\left[\ln \left(\frac{z}{z_{0}}\right)-\Psi_{m}(\varsigma)\right] \cdot\left[\ln \left(\frac{z}{z_{0 \theta}}\right)-\Psi_{h}(\varsigma)\right]}, \quad C_{H N}=\frac{\kappa^{2}}{\left[\ln \left(\frac{z}{z_{0}}\right)\right] \cdot\left[\ln \left(\frac{z}{z_{0 \theta}}\right)\right]}, \\
C_{Q}=\frac{\kappa^{2}}{\left[\ln \left(\frac{z}{z_{0}}\right)-\Psi_{m}(\varsigma)\right] \cdot\left[\ln \left(\frac{z}{z_{0 q}}\right)-\Psi_{q}(\varsigma)\right]} ., \quad C_{Q N}=\frac{\kappa^{2}}{\left[\ln \left(\frac{z}{z_{0}}\right)\right] \cdot\left[\ln \left(\frac{z}{z_{0 q}}\right)\right]} .
\end{gathered}
$$

where $C_{D N}, C_{H N}$, and $C_{Q N}$ are the exchange coefficients in neutral conditions. The exchange coefficients determined by equations $1.12-1.14$ are widely used in observational analyses and numerical simulations. Since the aerodynamic surface roughness is often estimated within the same MOS framework, to avoid complexity and redundancy, in practice, the exchange coefficients in neutral conditions can be estimated directly from the exchange coefficients corrected by stability. For example, equation 1.12 can be written as:

$$
\frac{1}{\sqrt{C_{D N}}}=\frac{1}{\sqrt{C_{D}}}+\frac{\Psi_{m}(\varsigma)}{\kappa},
$$

and subsequently, equation 1.15 is often used to calculate the neutral drag coefficient $C_{D N}$. 


\subsection{Scientific Questions}

As shown previously, the bulk transfer model can be closed by incorporating the MOS relationships into the parameterization framework. Incorporating MOS allows the exchange coefficients to be determined provided that the surface roughness and atmospheric stability are known. However, to appropriately use this framework to parameterize surface turbulent fluxes, there are issues that must also be addressed. Since the surface characteristics for ocean and land are completely different, issues pertaining to these differences in the exchange coefficients are reviewed separately in this section. For simplification, this study uses a SL under neutral conditions to illustrate the issues.

Over oceans, the surface waves depend on the wind speed so, the oceanic surface roughness is a function only of wind speed. Charnock (1955) first proposed that the roughness over the sea may be expressed as:

$$
z_{0}=\frac{\alpha u_{*}^{2}}{g},
$$

where $\mathrm{g}$ is gravity and $\alpha$ is an empirical coefficient, normally taken as 0.016 . Equation 1.16 has been widely used in observational analyses and numerical simulations. Combining equations 1.12 and 1.16 , and neglecting stability (a neutral SL is being considered), one can easily show that the neutral drag coefficient $C_{D N}$ increases with wind speed (the thick blue line in Figure 1.2). However, this assumption is not supported by observations. Figure 1.2 (adopted from Black et al. 2007) shows the observed neutral drag coefficients from multiple independent field experiments, where in most of the cases, $C_{D N}$ values in Figure 1.2 are computed from equation 1.15. It appears that $C_{D N}$ 
does not increase without limit as a function of wind speed; instead, it starts to level off above a set wind speed. Donelan et al. (2004) argued that such a behavior of $C_{D N}$ reflects the response of the ocean surface to the wind stress exerted on it. For low and moderate wind speeds, the aerodynamic roughness of the ocean surface, which is determined by roughness elements (or waves), increases with wind speed. However, the roughness elements become saturated at about $33 \mathrm{~ms}^{-1}$ (in their tank experiments) since the ocean surface reaches a maximum roughness in an aerodynamic sense. The argument by Donelan et al. (2004) simply suggests that the dependence of $C_{D N}$ on wind speed arises from the change of effective aerodynamic roughness in response to increasing winds. Consequently, the Charnock formula needs to be re-evaluated for higher wind speeds.

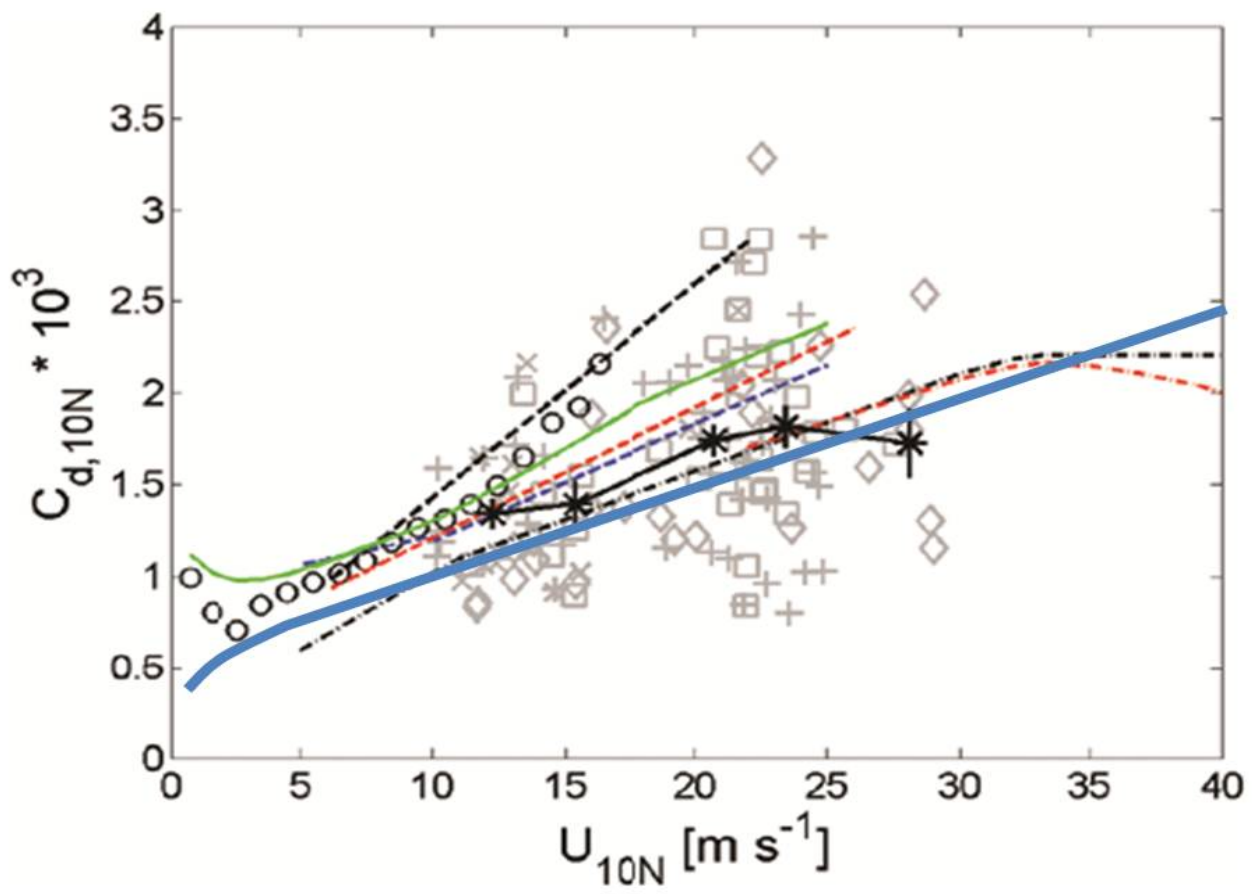

Figure 1.2 Plot adopted from Black et al. of observed $C_{D N}$ against 10-m wind speeds. Also shown is the theoretical line of $C_{D N}$ (thick blue). 
Another noticeable difference between observed and theoretical $C_{D N}$ is at low wind speeds. The theoretical line of $C_{D N}$ predicted by equations 1.12 and 1.16 curves toward smaller values at lower wind speeds. However, observations show that $C_{D N}$ increases dramatically with a decrease in wind speed when wind speeds become extremely small. The dramatic increase in $C_{D N}$ suggests that the response of the ocean surface to low wind speeds is quite different from the response at moderate or high wind speeds, and that the Charnock formula does not work as well at low wind speeds (i.e., it underestimates the ocean surface roughness). Regardless of the differences between observations and the theoretical prediction of $C_{D N}$ at low and high wind speeds, the evidence for different ocean surface responses to wind speeds suggests that the behavior of the drag coefficient can be well explained within the parameterization framework of the bulk transfer model and MOS, as long as the ocean surface roughness can be accurately determined. 


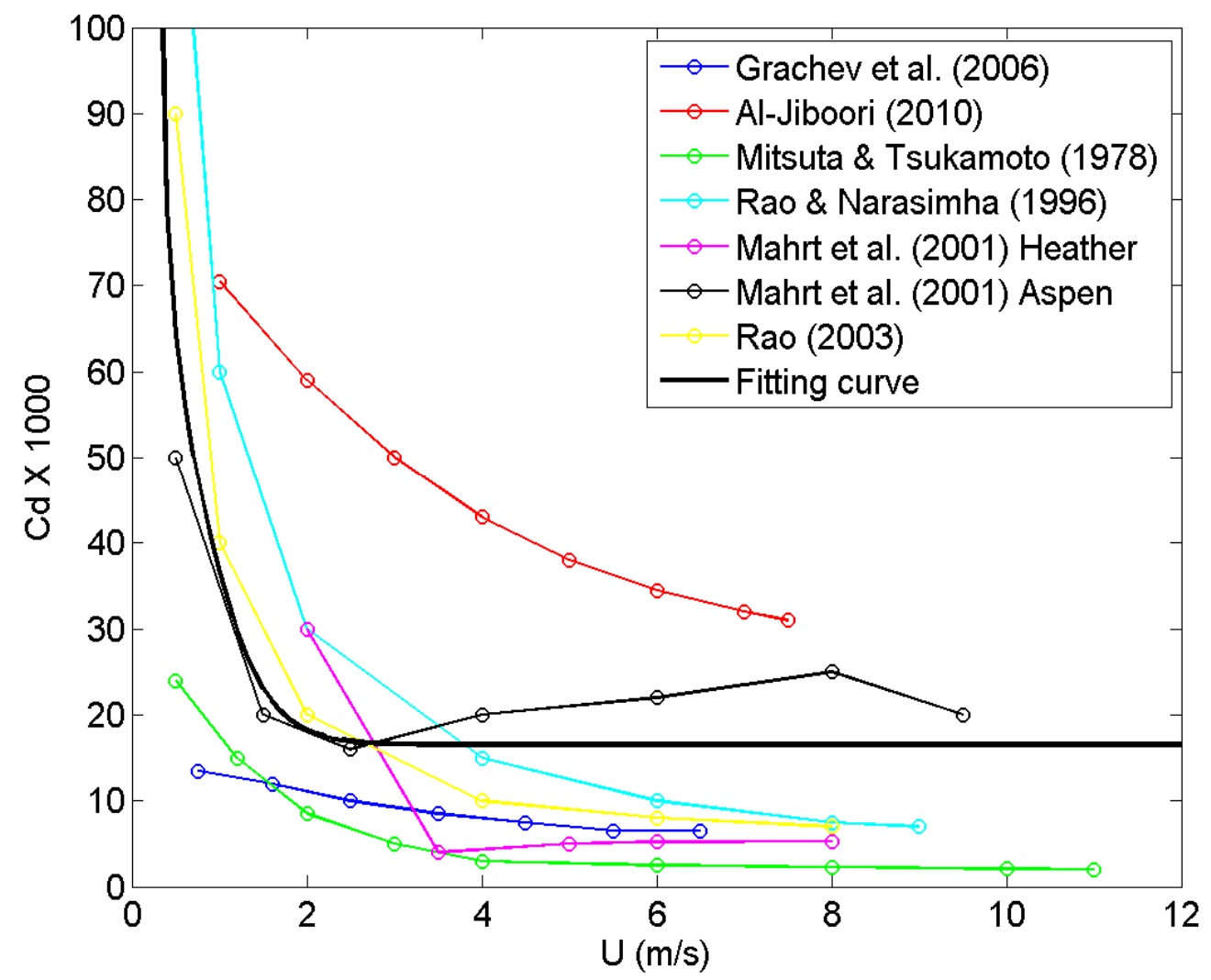

Figure 1.3 Plot of $C_{D N}$ against wind speed obtained from multiple field experiments over land.

The situation is quite different over land. One might expect that the surface roughness has little influence over land because of the static nature of the surface elements. As a result, MOS would predict a constant $C_{D N}$ that is independent of wind speed (the value of $C_{D N}$ will depend on the surface roughness). However this is not what observations show. Figure 1.3 shows $C_{D N}$ as a function of wind speed obtained from multiple independent field experiments over land. In some of the cases, $C_{D N}$ is calculated based on equation 1.15 , and in others, the drag coefficients are computed using 
near neutral data. For example, the criteria $|\varsigma|<0.05$ is used in Mahrt et al. (2001) and $|\varsigma|<0.15$ is used in the study by Mitsuta and Tsukamoto (1978). The thick black curve is the best fit curve of all the data points presented in the Figure 1.3. Although $C_{D N}$ from different field experiments exhibits a great deal of scatter, possibly due to large differences in surface roughness, all the data show a clear trend of $C_{D N}$ increasing with a decrease in wind speed for speeds below $3 \mathrm{~m} / \mathrm{s}$.

There have been attempts to explain such a variation of $C_{D N}$ as a function of wind speed within the MOS framework. For example, Mahrt et al. (2001) attributed such behavior of $C_{D N}$ to the increase in effective aerodynamic roughness with a decrease in wind speed. They argue that lower wind speeds enhance the viscous effects and reduce the streamlining of surface obstacles. The combined effect causes an increase in surface effective aerodynamic roughness as wind speed decreases.

It may be true that the streamlining of surface obstacles depends on wind, but the argument to attributing the observed variation of $C_{D N}$ solely to an apparent change in aerodynamic surface roughness, just like its maritime counterpart, must be further investigated. In fact, from equation 1.12 one can easily calculate the aerodynamic surface roughness for a given value of $C_{D N}$. Figure 1.4 shows the computed aerodynamic surface roughness that is required to obtain the $C_{D N}$ represented by the best fit curve in Figure 1.3. There is a sharp increase of $z_{0}$ for wind speeds lower than $2 \mathrm{~m} / \mathrm{s}$, and its magnitude can reach up to $10 \mathrm{~m}$ for extremely low wind speeds. It is difficult to provide a physical explanation for such a sharp increase of $z_{0}$ considering the static nature of the 
land surface roughness elements. Therefore, one of the objectives of this thesis is to revisit the issues of drag coefficient in the low wind regime, provide a physically sound explanation for the observed variation of drag coefficient, and attempt to extend the classic MOS framework into the low wind regime.

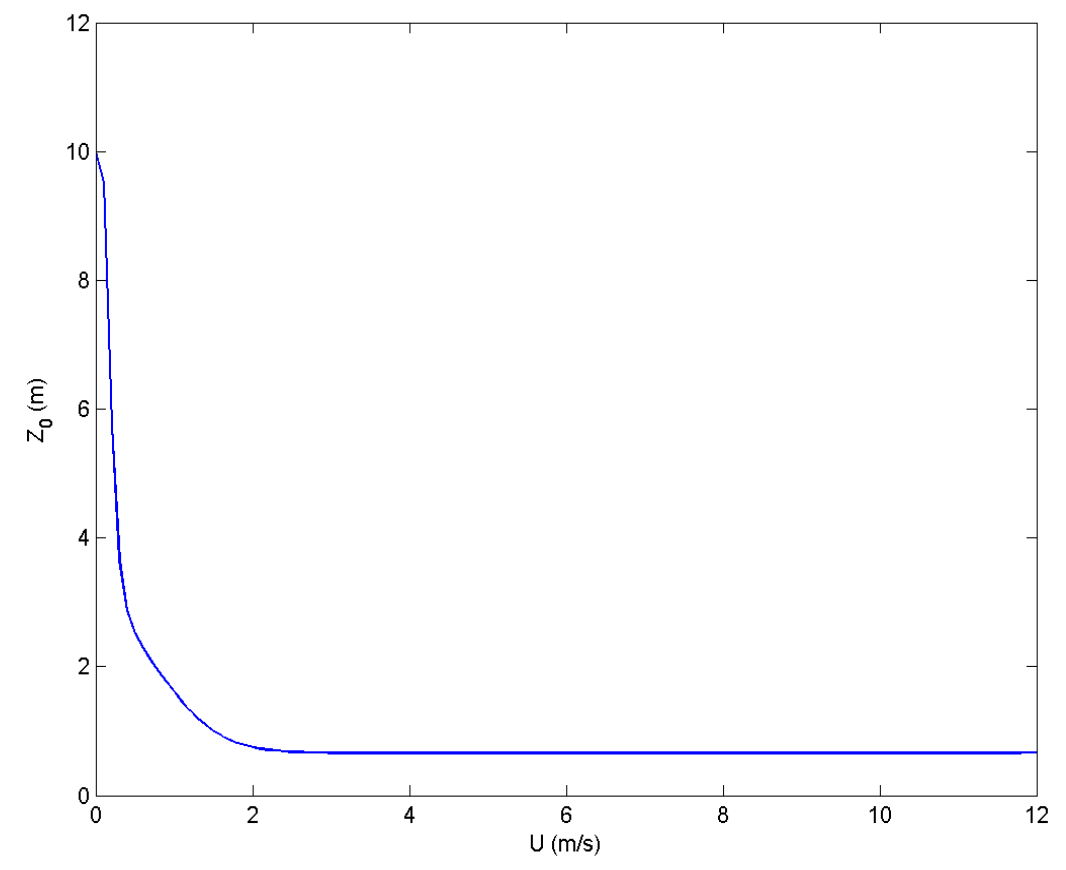

Figure 1.4 Plot of the computed aerodynamic surface roughness against wind speed that is required to obtain a given $C_{D N}$ value represented by the best fit curve in Figure 1.3.

Compared with the relatively intense studies on drag coefficient, the exchange coefficients for heat and moisture are less well documented because of the difficulties in obtaining high resolution temperature and moisture observations. In previous studies, almost all relevant high resolution SL observations were collected in fair weather conditions since precipitation often causes sensor malfunctions, further preventing an accurate estimation of turbulent fluxes using the eddy correlation method. Although 
precipitation continues to be an obstacle for obtaining accurate measurements of temperature and moisture, this problem has been largely alleviated in recent years due to advancements in instrumentation. For example, the newly developed LICOR LI-7200 enclosed $\mathrm{CO}_{2} / \mathrm{H}_{2} \mathrm{O}$ gas analyzer maximizes the strengths of traditional open-path instruments (good frequency response) and closed-path instruments (minimal data loss due to precipitation). The new GILL WindMaster Pro (WMP) sonic anemometer can operate properly in up to $300 \mathrm{~mm} / \mathrm{hr}$ precipitation conditions. This thesis presents data collected by the LI-COR LI-7200 gas analyzer and Gill WMP sonic anemometer in all weather conditions, including harsh precipitating conditions, and characterizes $C_{H}$ and $C_{Q}$ in terms of wind speed and atmospheric stability. 


\section{OBSERVATIONS}

\subsection{Instrumentation}

\subsubsection{IHRC 10-meter PWT}

Tower based instrumentation systems have proven to be a powerful tool in detecting turbulent motions in the ABL. The portable wind tower (PWT) used in this study was designed and constructed by the International Hurricane Research Center (IHRC) for use in the Florida Coastal Monitoring Program (FCMP). The original goal of the FCMP was to better understand surface wind fields during hurricane landfall and their resultant impact on residential structures. Figure 2.1 shows the current configuration of the PWT. The tower mast is built onto a trailer and powered by a set of three oversized $12 \mathrm{~V}$ batteries making it easy to transport and deploy. It stands 10 -meters tall, weighs approximately $7000 \mathrm{lbs}$, and is capable of withstanding a $90 \mathrm{~m} / \mathrm{s}$ wind gust, corresponding to a Category 5 hurricane on the Saffir-Simpson Hurricane Wind Scale. Two sets of 3-D Gill propeller anemometers and an RM Young wind vane anemometer were originally installed at $5 \mathrm{~m}$ and $10 \mathrm{~m}$. Both instruments can record wind velocities at a frequency of $10 \mathrm{~Hz}$. The PWTs have been successfully deployed in multiple landfalling tropical cyclones over the past several hurricane seasons. 


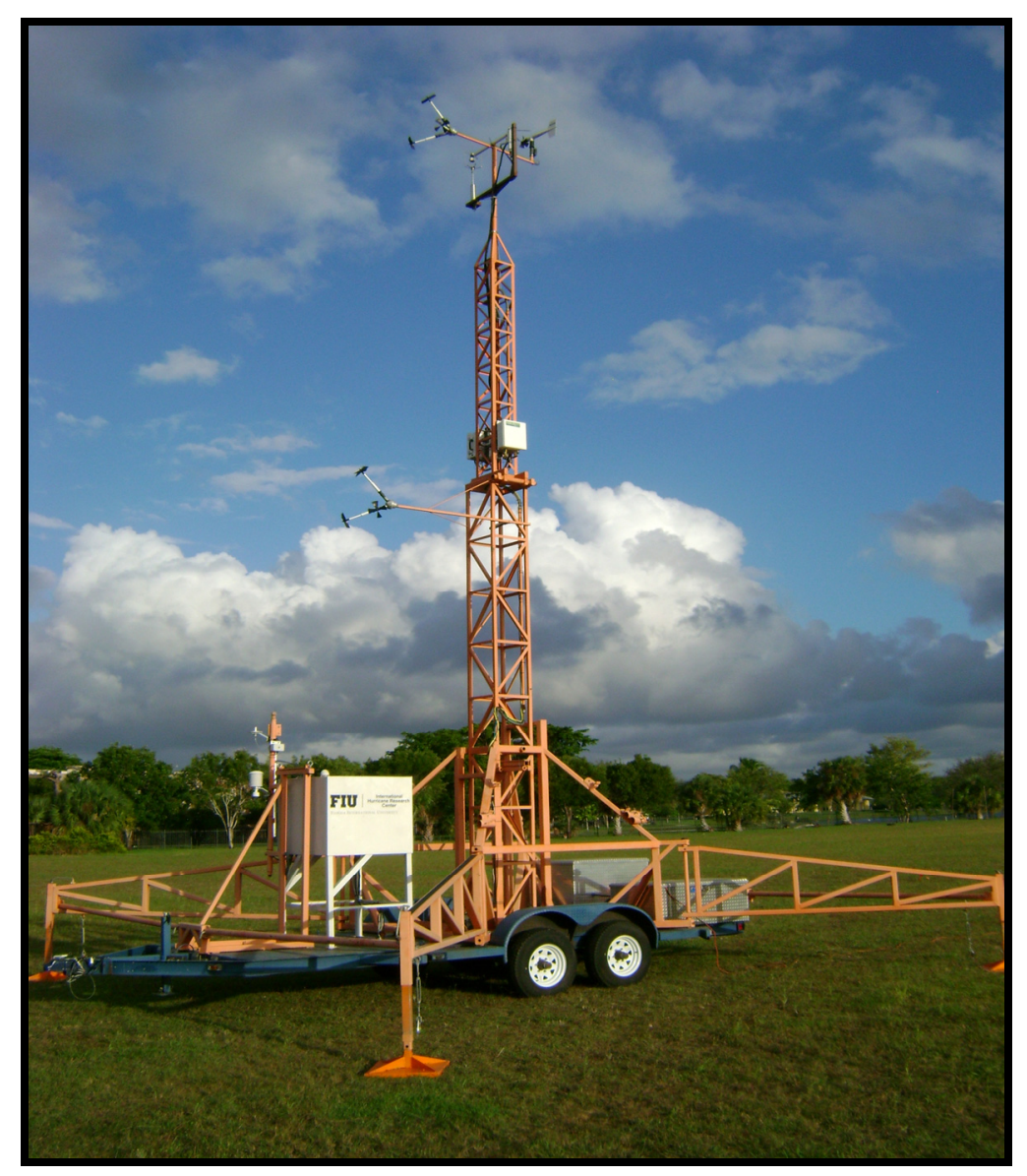

Figure 2.1 Photo of the PWT set up behind the FIU Engineering Campus.

\subsubsection{Eddy covariance observing system}

In the summers of 2011 and 2013, the instruments on the tower were upgraded by adding two sets of Gill WMP sonic anemometers and LI-COR LI-7200 enclosed $\mathrm{CO}_{2} / \mathrm{H}_{2} \mathrm{O}$ gas analyzers at $5-\mathrm{m}$ and $10-\mathrm{m}$. Figure 2.2 provides a view of the upgraded eddy covariance observing system at 10-m, which consists of a sonic anemometer, a gas analyzer, a propeller anemometer, and a wind vane monitor. 


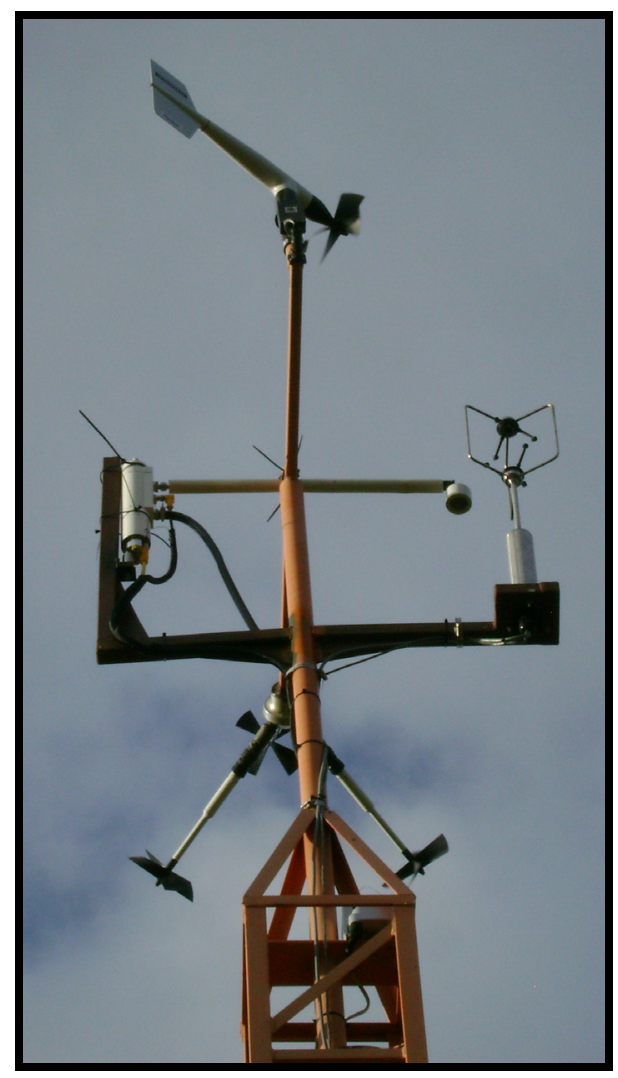

Figure 2.2 Photo of the sonic anemometer (right) and gas analyzer (left) atop the PWT. Also shown in the photo are the pre-existing propeller anemometers (bottom) and wind vane monitor (top).

The Gill WMP sonic anemometer measures the three dimensional components of the wind: $\mathrm{u}$, an along wind component; $\mathrm{v}$, a cross wind component; and $\mathrm{w}$, a vertical component. The sonic anemometer works by measuring the difference in transit time of ultrasonic pulses transmitted in both directions between a pair of transducer heads. The transit time depends on the speed of sound and on the velocity of the air along its path. The path length between the pairs of heads is known, and when combined with a specific transit time, a velocity is measured. Furthermore, the speed of sound is dependent on air density, which in turn depends on temperature and water vapor mixing ratio. Utilizing 
these principles, the sonic anemometer also provides a sonic temperature, $T S$, approximately equivalent to the atmospheric virtual temperature. $T s$ is computed by:

$$
T S=T \cdot\left(1+\left(\frac{\gamma_{v}}{\gamma_{d}}-\frac{M_{v}}{M_{d}}\right) \cdot \frac{e}{p-\left(1-\frac{M_{v}}{M_{d}}\right) \cdot e}\right) .
$$

Above, $T$ is the ambient temperature, $\frac{\gamma_{v}}{\gamma_{d}}$ is the ratio of specific heats of vapor and dry air, $\frac{M_{v}}{M_{d}}$ is the ratio of the molar masses for water vapor and dry air, $e$ is the water vapor pressure and $p$ is the total air pressure (Lazinger at al. 2005). The WMP sonic anemometer has a maximum operating wind speed of $65 \mathrm{~ms}^{-1}$ and a data output rate of up to $32 \mathrm{~Hz}$. The specifications for the Gill WMP are included in appendix A.

The LI-7200 closed path infrared gas analyzer computes a range of variables. The notable ones pertaining to this research include: concentration density of water vapor, dew point temperature, total atmospheric pressure, and various other diagnostic values. The principle of operation for the gas analyzer is the measurement of atmospheric trace gases by determining the absorption of an infrared beam emitted within the optical path of the sensor. The beam is absorbed by a specific gas $\left(\mathrm{H}_{2} \mathrm{O}\right.$ or $\left.\mathrm{CO}_{2}\right)$ at a certain wavelength, reducing the intensity of the infrared beam. The reduction in intensity, as a function of the gases' molar concentration, is measured by the sensor (Aubient 2013: 40). The molar concentration of the respective gas can then be converted into (for the case of $\mathrm{H}_{2} \mathrm{O}$ ) mixing ratio. The output variable labeled " $\mathrm{H}_{2} \mathrm{O}$ concentration density" can be 
converted to water vapor mixing ratio by simply multiplying by the ratio of molar masses for water vapor and dry air, $\frac{M_{v}}{M_{d}}$ (LI-COR Biosciences 2010).

Gas (air) inside the optical path of the LI-7200 is continually recycled via a flow module (pump) providing a continuous, but self regulating, supply of ambient air to the analyzer. An intake tube is connected to the inlet port of the gas analyzer. The opposite end is positioned directly adjacent to the WMP to sample the same air that the WMP is measuring (see Figure 2.2). The rate of flow into the intake tube and through the analyzer is measured in Standard Liters Per Minute (SLPM) and is adjustable by the user. For this specific research we set the flow to 14 SLPM, the recommended level stated in the LI-7200 user manual. The specifications for the LI-7200 are included in appendix A.

The data acquisition system for data collected by the tower instruments is housed in a small white box near the base of the tower. All the instruments including the electronic components inside the white box are powered by three large $12 \mathrm{~V}$ batteries located at the left rear of the tower in a silver waterproof container. There were inconsistency issues regarding power requirements of each eddy covariance set up, so to further avoid any complication with power distribution throughout the instruments on the tower, a pair of simple switches was installed. The switches are installed on the power cable that provides power for the gas analyzer/flow module system. These two instruments consume the most power and therefore are wired directly to the battery bank, bypassing any voltage converters, thus ending any further issues with power consumption. Data collected by the instruments are transmitted via ethernet cable, logged using software provided by LI-COR, and is saved as 15 minute tab delimited ASCII files 
onto the hard drive of a laptop located inside the white control box on the tower. For this research the sampling rate of the instruments is set to $20 \mathrm{~Hz}$.

Accompanying the eddy covariance observing systems is a set of ground temperature sensors (Figure 2.3). Each sensor consists of a thermistor connected via telephone cable to a small blue box. The blue box houses a breadboard with soldered resistance bridges to convert changes in thermistor resistance into voltages. These voltage changes are digitized and logged via a DATAQ datalogger (the other blue box in Figure 2.3) using the software provided with the datalogger. The whole ground temperature system is powered by a $9 \mathrm{~V}$ battery. The thermistors are calibrated using a water bath test in a controlled and monitored indoor environment. The coefficients derived from the water bath test are applied when converting the raw voltage outputs units of degrees Celsius. During a deployment, the ground temperature sensors are simply placed on the surface of the ground at the base of the tower. They contain weatherproof casing around their sensitive parts. 


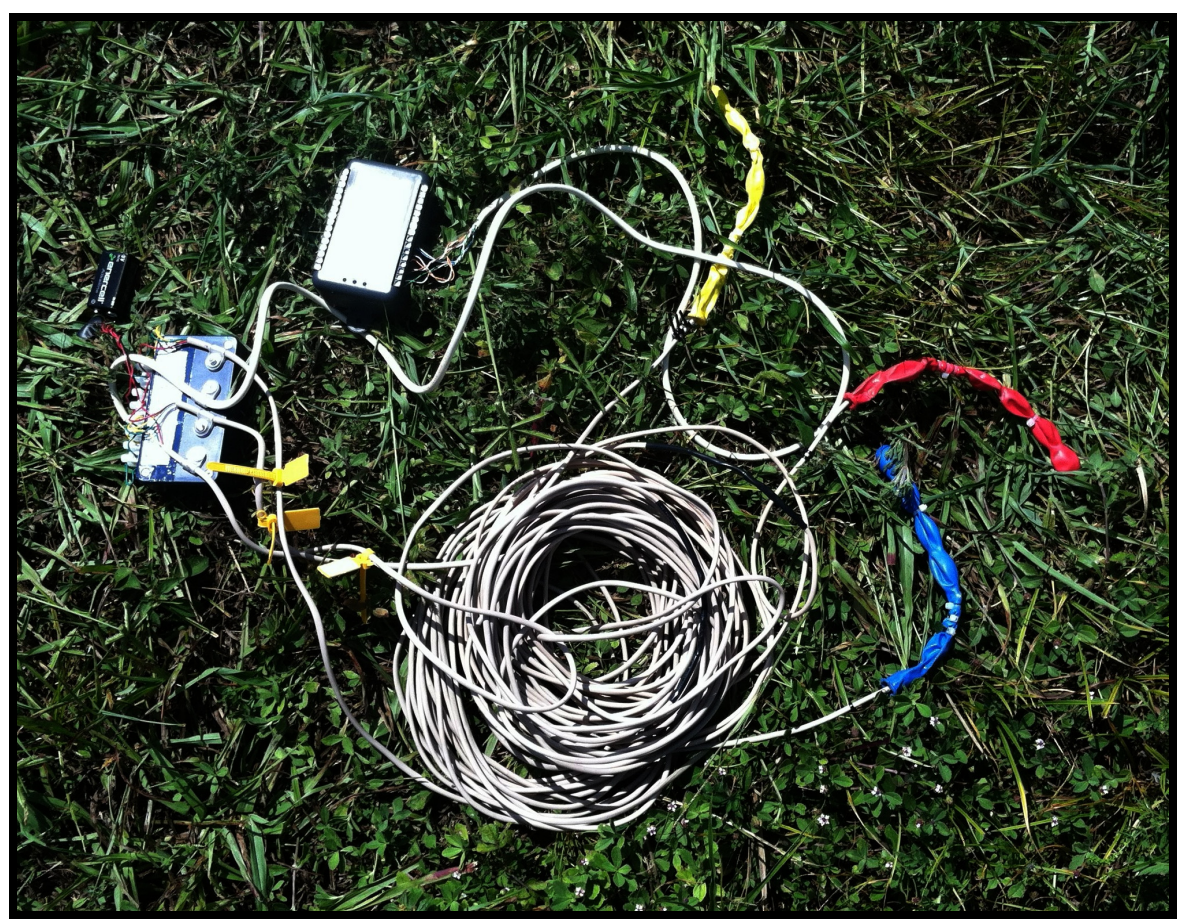

Figure 2.3 Photo of the ground temperature sensors

\subsection{Tower Deployments}

In the summer of 2012, the tower was deployed at several coastal locations under different weather conditions. The tower sites and weather conditions are summarized as follows.

\subsubsection{Everglades National Park - Lake Chekika Deployment}

The PWT was deployed at Lake Chekika in Everglades National Park (ENP) during the period of October 12 through October 16, 2012. Figure 2.4 shows the tower location for the ENP deployment. The tower was set up in a small parking lot, which was almost entirely under water during the experimental period. From the vantage point atop the tower, there is a significant amount of standing water in all directions, which is 
typical during mid-October. Aside from a small patch of larger vegetation and a few trees immediately to the north of the tower, the surface is surrounded in all other directions by a nearly homogenous covering of sawgrass wetland, with very few large surface roughness elements. The sawgrass stalks have an average height of $\sim 1 \mathrm{~m}$. The period of observations at the ENP site represents 4 diurnal cycles with occasional showers.

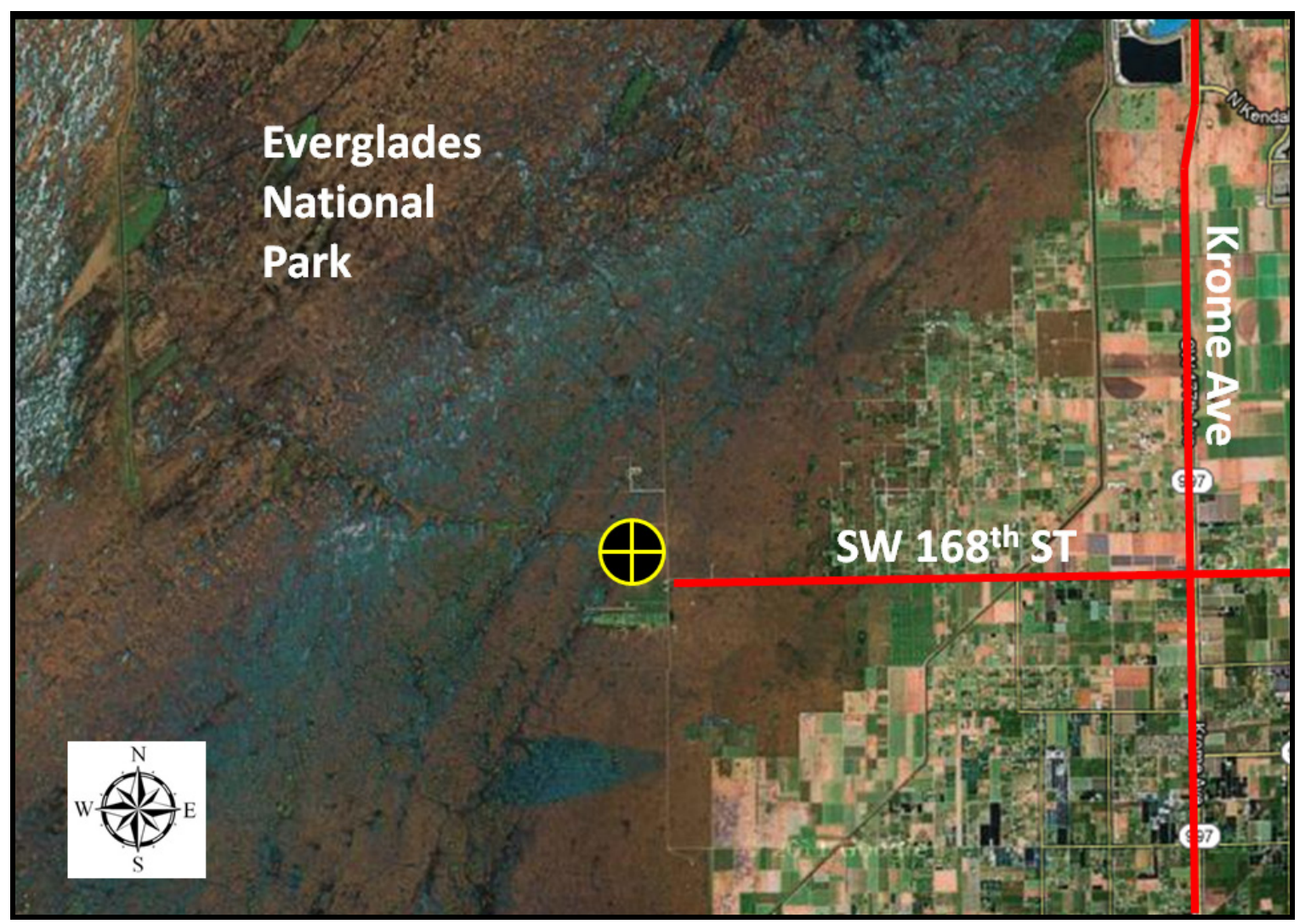

Figure 2.4 Aerial view of the PWT deployment location at the ENP site. The small yellow and black target symbol is the location of the tower when it was deployed. 


\subsubsection{Biscayne Bay Deployment}

The PWT was deployed during the time period of October $21^{\text {st }}$ through October $27^{\text {th }}$ at the FIU Biscayne Bay Campus (BBC). This site was chosen because of its relatively easy access and its location, which is directly adjacent to Biscayne Bay and $\sim 1$ mile from the Atlantic Ocean (Figure 2.5). The ENP site contrasts the BBC site in that it is completely isolated from any structural development. The general surroundings of the tower's location consists of a variety of roughness elements including water to the south and southeast, and small bushes, shrubs, and some taller trees in all other directions. The immediate surrounding at the base of the tower is a slightly elevated grass covered field approximately $200 \mathrm{~m}$ by $75 \mathrm{~m}$.

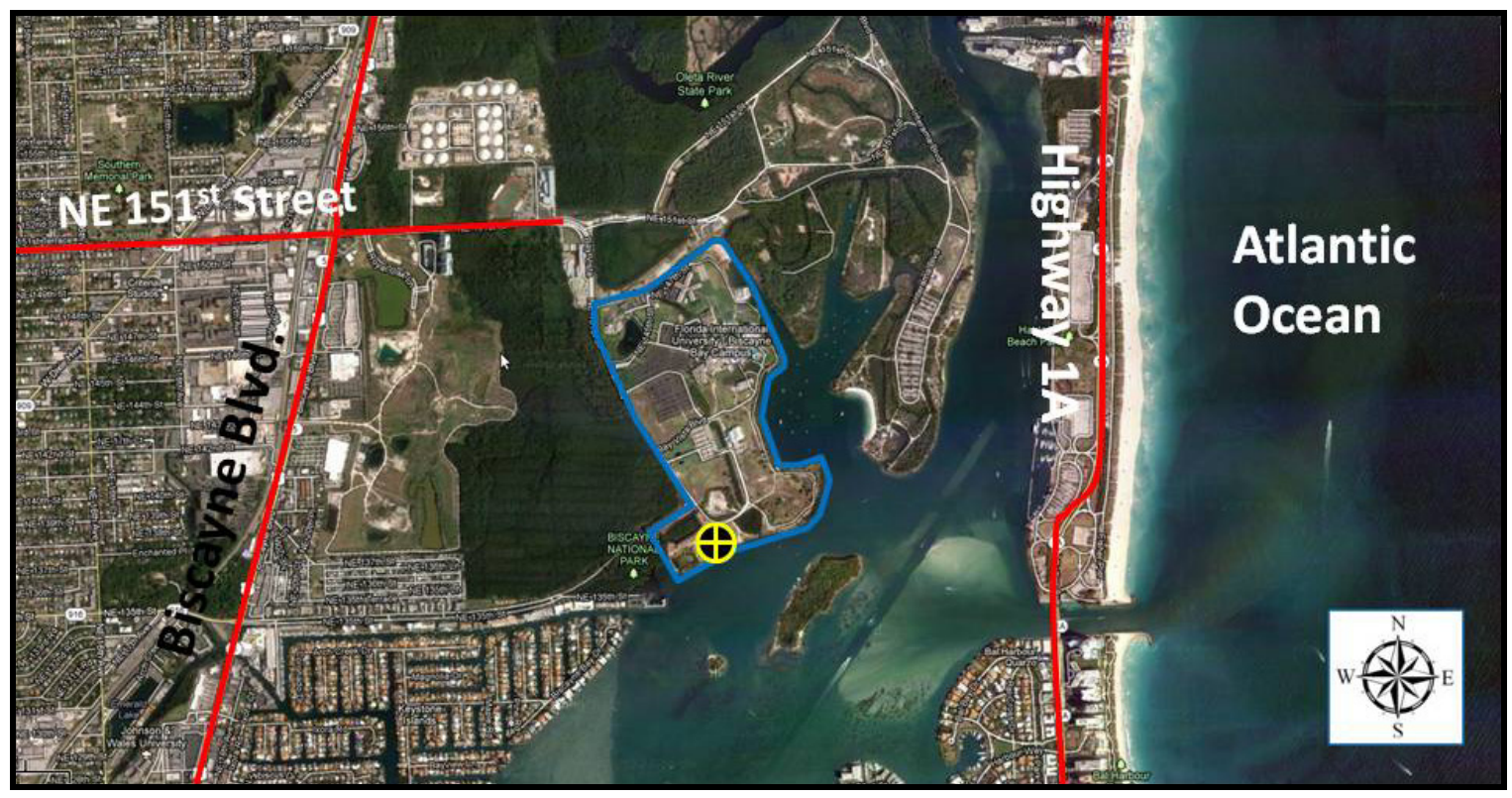

Figure 2.5 Aerial view of the PWT deployment location at BBC. The blue outline is $\mathrm{BBC}$ and the small yellow and black target symbol is the location of the tower when it was deployed. 
The period of observation at the BBC site includes 6 diurnal cycles, during which Hurricane Sandy passed to the east of Florida within 200 miles of the tower location. Figure 2.6 shows a visible satellite image of Sandy as it passed over the northern Bahamas.

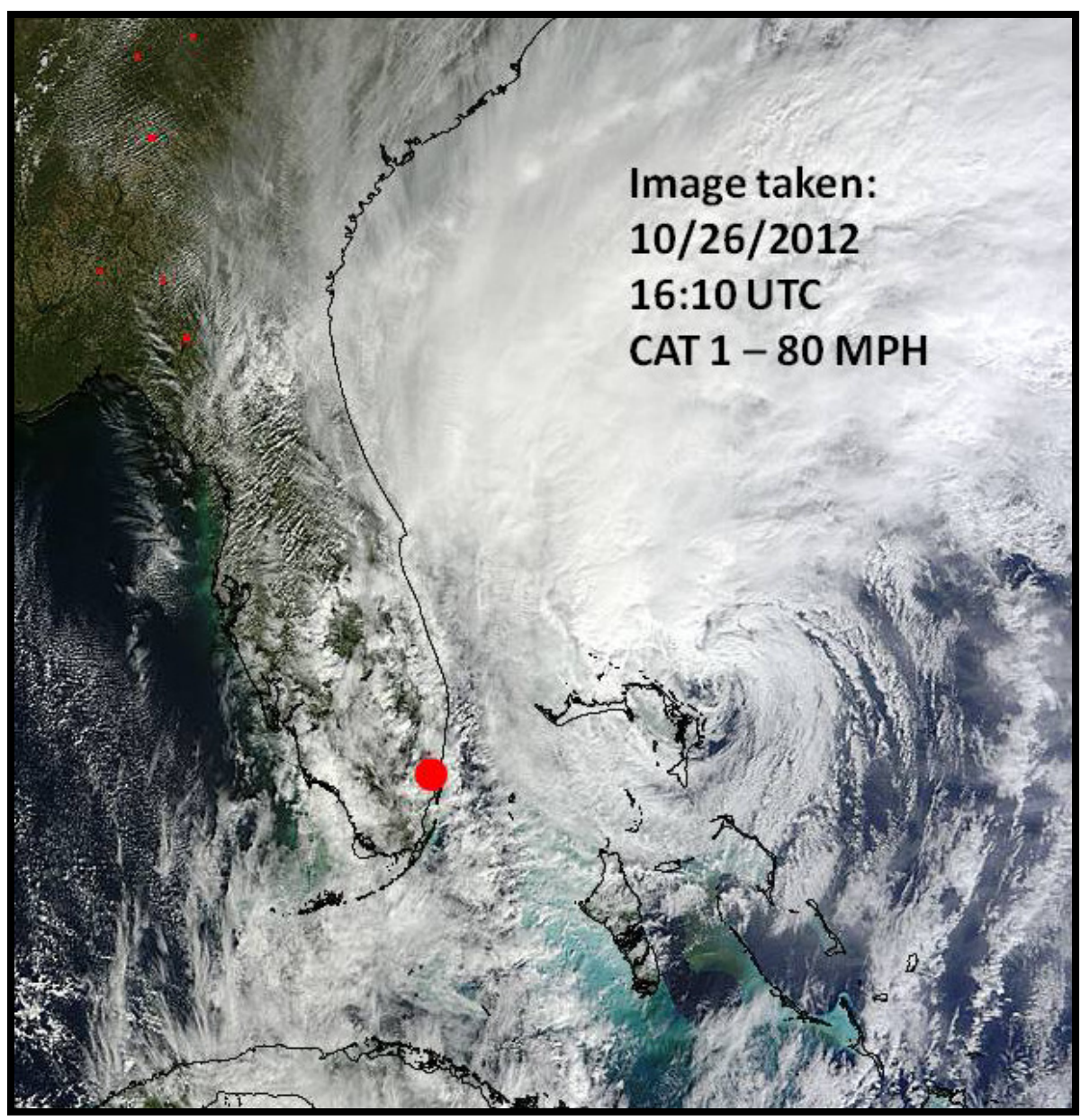

Figure 2.6 Satellite image of Hurricane Sandy passing by the Florida peninsula. The red dot indicates the location of the tower at BBC.

\subsubsection{Naples Municipal Airport - Tropical Storm Isaac}

The PWT was deployed at the Naples Municipal Airport in Naples, FL (Figure

2.7) from 19:45 EDT August $25^{\text {th }}$ to $17: 30$ EDT August $26^{\text {th }}$, a time period during which 
Tropical Storm Isaac passed near Key West, FL. The tower location was within the outer rain bands of Isaac, which were oriented mostly in the North - South direction and moved from East to West. The 15 minute averaged wind speeds measured by the PWT are just below tropical storm force. The surface surrounding the tower is a flat landscape (a mix of concrete paving and grass) for several hundred meters in all directions.

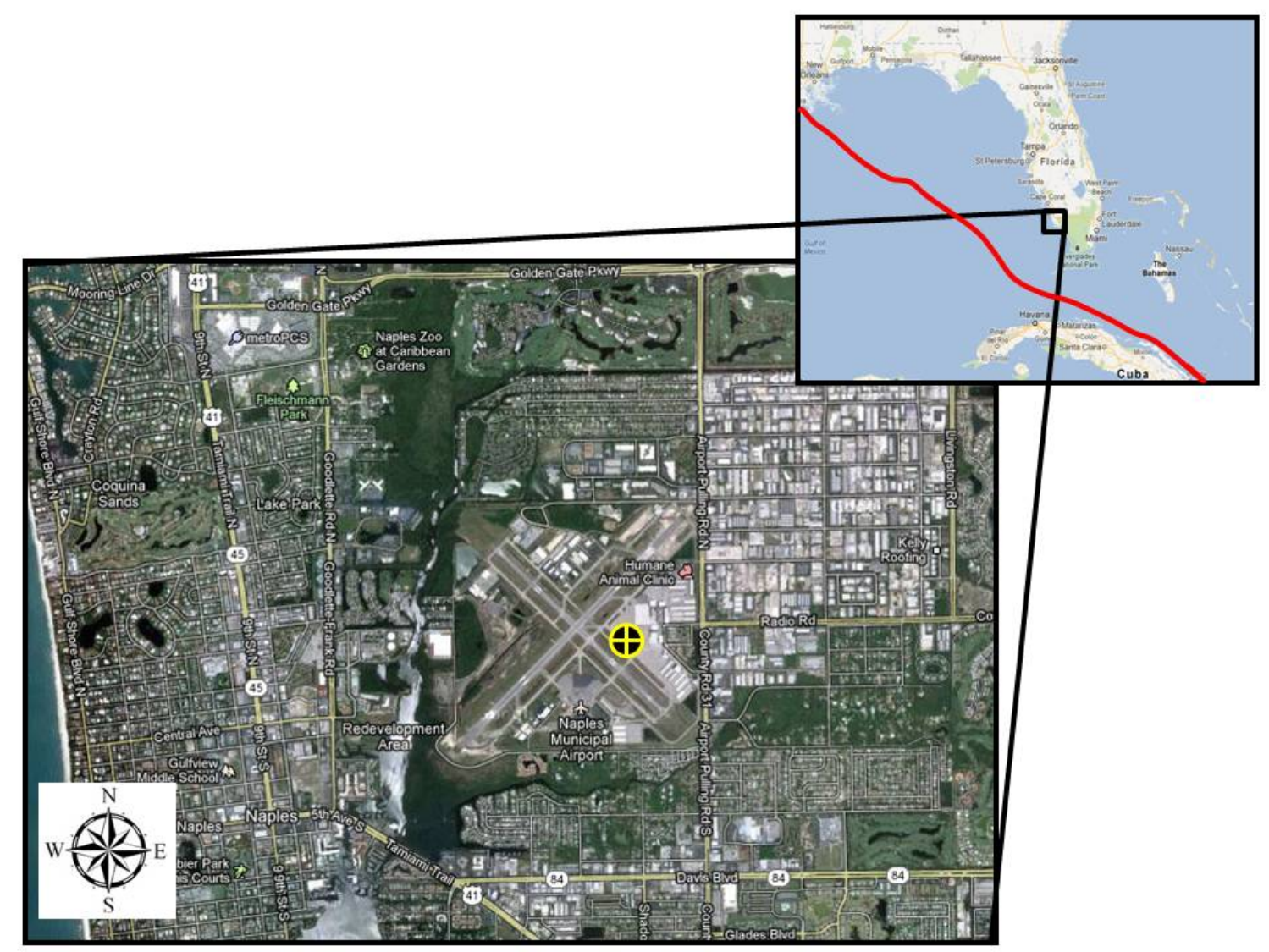

Figure 2.7 Aerial view of the PWT deployment location at the NMA site. The red line in the upper map is an approximation to the track of Tropical Storm Isaac.

\subsubsection{Waveland, Mississippi - Tropical Storm/Hurricane Isaac}

After deploying the PWT at the Naples site, we made a second deployment in Waveland, MS (Figure 2.8) as Isaac made landfall near the mouth of the Mississippi 
River. The site contains no structures or vegetation in any direction from the tower for approximately $200 \mathrm{~m}$. The tower was in the range of both Kevin Knupp's Doppler radar at the Picayune Airport and the Slidell WSR-88D radar. The data collection started at approximately 11:00 CDT August $28^{\text {th }}$ when Isaac was located just a few hundred miles offshore with maximum sustained winds of 61 knots. Isaac finally reached hurricane status just before landfall at approximately 1300 CDT August $28^{\text {th }}$. Subsequently, Hurricane Isaac tracked further west making landfall twice along the Louisiana coast. At approximately $0800 \mathrm{CDT}$ August $29^{\text {th }}$, the center of Hurricane Isaac was located directly over Houma, Louisiana, exactly 93 miles from the location of the tower in Waveland. Therefore, the maximum sustained wind speeds the tower recorded fell short of hurricane strength. The data collection was terminated at $1600 \mathrm{CDT}$ August $30^{\text {th }}$. 


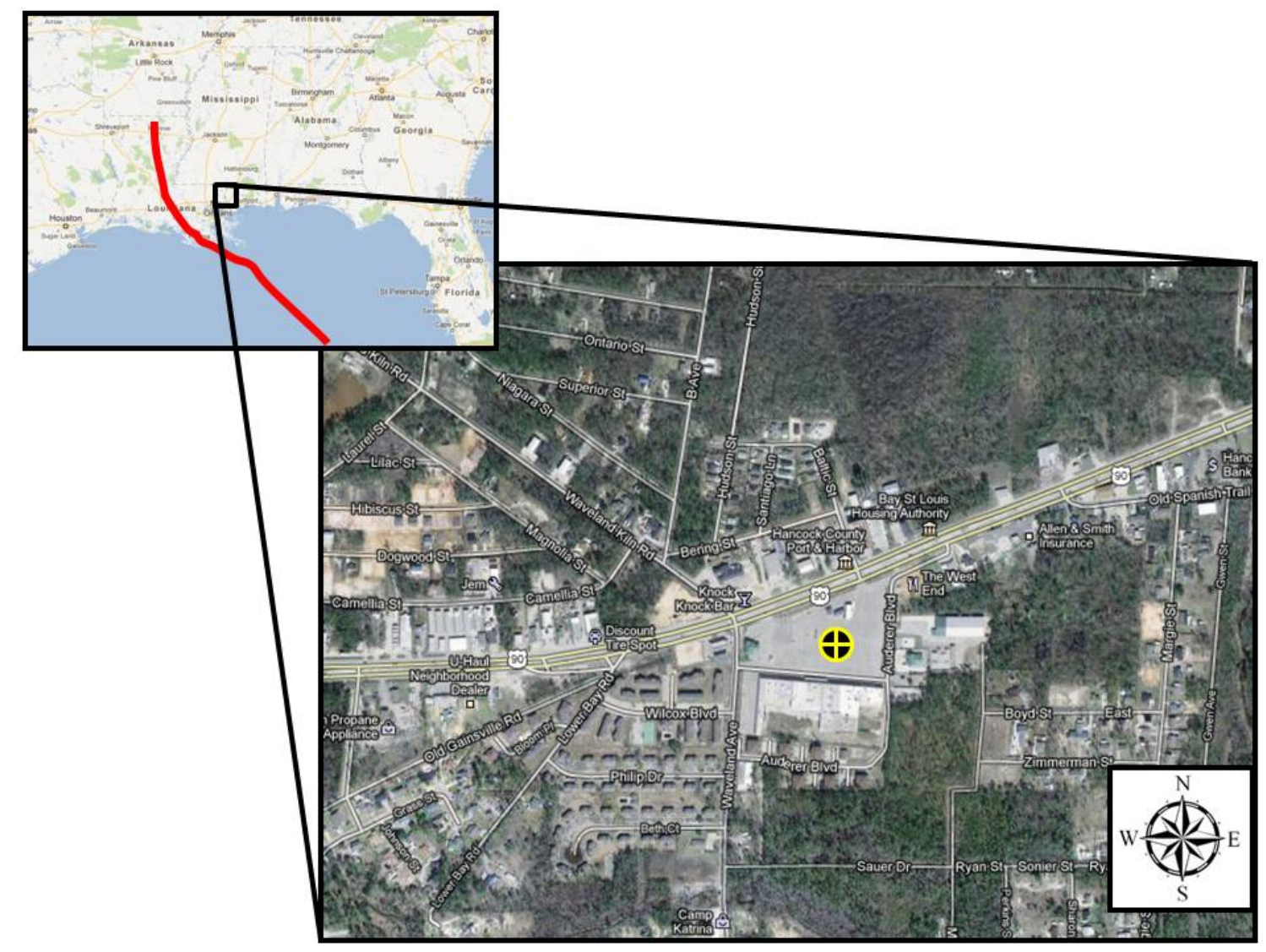

Figure 2.8 Aerial view of the PWT deployment location at the Waveland site. The red line in the upper map is an approximation to the track of Tropical Storm/Hurricane Isaac. 


\section{DATA ANALYSIS AND METHODS}

\subsection{Eddy Correlation Method}

The eddy correlation method dates back to Montgomery (1948), where he proposed a method for calculation of the exchange of heat, mass, and momentum between the surface and overlying atmosphere. One requirement for an accurate estimation of higher order turbulent moments is that the meteorological sensors used in a given study must be fast responding, and record at frequencies of at least $10 \mathrm{~Hz}$, so that all energy containing eddies in the turbulent spectrum can be resolved. In other words, all eddies with a size greater than the inertial sub-range must be resolved. In this study, all data are collected at a frequency of $20 \mathrm{~Hz}$.

One of the first steps in the eddy correlation method is to use the Reynolds decomposition method to decompose the instantaneous data value into its mean and turbulent components:

$$
a^{\prime}=a-\bar{a}
$$

In equation 3.1, $a$ is the instantaneous value of a given variable, and the prime and overbar indicate the perturbation and mean, respectively. In this study, a 15 minute time interval is used for calculating the mean. In practice, since data in a 15-minute long period may contain an apparent trend, which will produce strong red noise in the spectrum, a de-trending process is also applied to remove any linear trend present. Once the turbulent components are determined, the variance and covariance are computed through: 


$$
\begin{gathered}
\overline{a^{\prime 2}}=\frac{1}{N} \sum_{i=0}^{N-1} a_{i}^{\prime 2}, \\
\overline{a^{\prime} b^{\prime}}=\frac{1}{N} \sum_{i=0}^{N-1} a_{i}^{\prime} b_{i}^{\prime} .
\end{gathered}
$$

This study uses the eddy correlation method to compute kinematic momentum, heat, and moisture fluxes, $\overline{u^{\prime} w^{\prime}}, \overline{v^{\prime} w^{\prime}}, \overline{u^{\prime} v^{\prime}}, \overline{w^{\prime} \theta^{\prime}}$, and $\overline{w^{\prime} q^{\prime}}$, as well as velocity, potential temperature, and mixing ratio variances, $\overline{u^{\prime 2}}, \overline{v^{\prime 2}}, \overline{w^{\prime 2}}, \overline{\theta^{\prime 2}}$, and $\overline{q^{\prime 2}}$. Subsequently, the turbulent kinetic energy (TKE) is estimated as:

$$
e=0.5 *\left(\overline{u^{\prime 2}}+\overline{v^{\prime 2}}+\overline{w^{\prime 2}}\right)
$$

\subsection{Quality Control and Assurance Procedures}

\subsubsection{Spike Removal}

The use of high resolution sonic anemometer and gas analyzer instruments requires quality control of the observed data to remove erroneous data. Often, sonic anemometer data contains distinct spikes caused by rain droplets, birds, insects, feces, and other unknown reasons. In this study, the method proposed by Schmid et al. (2000) is used to remove all spikes. A data point is defined as a spike if the following condition is met:

$$
\left|\chi_{i}-\bar{\chi}_{j}\right| \geq D \cdot \sigma_{j} \rightarrow \text { spike }
$$

In the above equation, $\chi$ represents a generic variable; the subscript $j$ denotes the $j^{\text {th }}$ averaging time period $(15 \mathrm{~min}) . \sigma_{j}$ is the standard deviation of the $j^{\text {th }}$ averaging time period. The subscript $i$ denotes the $i^{\text {th }}$ observation in the $\mathrm{j}^{\text {th }}$ averaging time period. $D$ is a 
discrimination factor that depends on the variable being assessed. Given the general behavior of high resolution $20 \mathrm{~Hz}$ measurements of temperature and water vapor versus the 3-D wind components, a value of $\mathrm{D}=4.0$ is assigned for temperature and moisture, and $\mathrm{D}=5.0$ is assigned for the wind velocity components.

\subsubsection{Sonic Anemometer Tilting Correction}

Another necessary quality control process is to apply a tilt correction algorithm to the sonic anemometer output. Studies show that large errors in estimating momentum fluxes can occur due to small errors in the alignment of turbulent wind sensors (e.g., Pond, 1968; Deacon, 1968; Kaimal and Haugen, 1969; Dyer and Hicks, 1972; Dyer, 1981). The source of large momentum flux errors can be attributed to the cross contamination of velocities that occur in a tilted sensor, such that fluctuations in the longitudinal components of the wind appear as vertical velocity fluctuations, and vice versa. In fact, tilting cannot be completely avoided because of the presence of slight slopes in the terrain surrounding a tower, as well as difficulties associated with precisely leveling the sensor. Wilczak et al. (2001) showed that for a $1^{\circ}$ tilt in a sonic anemometer, the estimation error of momentum fluxes is typically greater than $10 \%$ under moderately unstable conditions, but can be as large as $100 \%$ under strong convective conditions.

One common method to minimize the error in estimating fluxes due to tilting is to rotate the sonic anemometer coordinate into a streamwise coordinate, which involves a series of two rotations. This method, often named the Double Rotation (DR) method, was first proposed by Tanner and Thurtell (1969). In a typical right-hand local Cartesian coordinate framework, there are three degrees of freedom available that allow three 
rotations around the $z$-axis, the new $y$-axis, and the new $x$-axis, respectively, resulting in the Euler angles $\alpha, \beta$, and $\gamma$. These rotations can be expressed mathematically in matrix form as:

$$
R_{01}=\left[\begin{array}{ccc}
\cos \alpha & \sin \alpha & 0 \\
-\sin \alpha & \cos \alpha & 0 \\
0 & 0 & 1
\end{array}\right], \quad R_{12}=\left[\begin{array}{ccc}
\cos \beta & 0 & \sin \beta \\
0 & 1 & 0 \\
-\sin \beta & 0 & \cos \phi
\end{array}\right], \quad R_{23}=\left[\begin{array}{ccc}
1 & 0 & 0 \\
0 & \cos \gamma & \sin \gamma \\
0 & -\sin \gamma & \cos \gamma
\end{array}\right]
$$

The above rotations (3.6) are applied in order to convert the wind vector in the sonic anemometer coordinate frame into the wind vector in the mean streamwise coordinate frame to yield:

$$
\left(\begin{array}{c}
\bar{u}_{3} \\
\bar{v}_{3} \\
\bar{w}_{3}
\end{array}\right)=R_{03}(\alpha, \beta, \gamma)\left(\begin{array}{c}
\bar{u}_{0} \\
\bar{v}_{0} \\
\bar{w}_{0}
\end{array}\right), \quad R_{03}(\alpha, \beta, \gamma)=R_{23}(\gamma) \cdot R_{12}(\beta) \cdot R_{01}(\alpha)
$$

For the scale flux matrix, it gives:

$$
\left(\frac{\overline{u_{3}^{\prime} x^{\prime}}}{\frac{v_{3}^{\prime} x^{\prime}}{w_{3}^{\prime} x^{\prime}}}\right)=R_{03}(\alpha, \beta, \gamma)\left(\frac{\overline{u_{0}^{\prime} x^{\prime}}}{\overline{v_{0}^{\prime} x^{\prime}}}\right)
$$

and for the wind component (co)variance matrix, it gives:

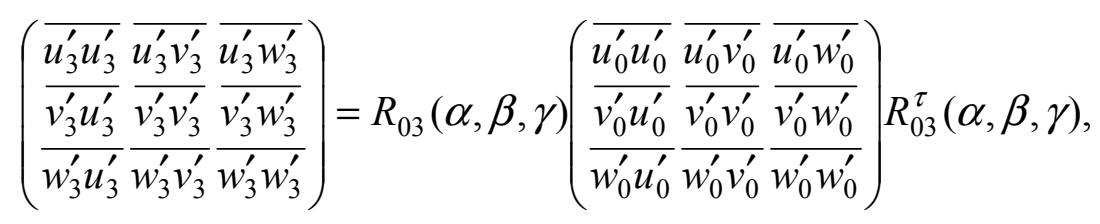

where, $R_{03}^{\tau}(\alpha, \beta, \gamma)$ is the transpose of $R_{03}(\alpha, \beta, \gamma)$.

In the DR correction, the final vector orientation is as follows: the $z$-axis is normal to and points away from the mean local streamline (15-minute average used in this study), while the $x$-axis is streamwise to the mean flow with $x$ increasing in the direction 
of the flow. To obtain the desired vector basis, the first rotation is performed to align $\bar{u}$ into the mean wind direction and force $\bar{v}=0$, resulting in the yaw angle $\alpha$ :

$$
\alpha_{D R}=\tan ^{-1}\left(\frac{\bar{v}_{0}}{\bar{u}_{0}}\right) .
$$

The second rotation is performed to minimize $\bar{w}$. This rotation is based on the assumption that the mean vertical velocity in the SL over a sufficiently long time period is zero. The second rotation yields the pitch angle $\beta$

$$
\beta_{D R}=\tan ^{-1}\left(\frac{\bar{w}_{1}}{\bar{u}_{1}}\right) .
$$

In the DR correction, the third angle $\gamma$ in equation 3.6 is simply zero. By utilizing equations 3.6 - 3.11, one can easily calculate the corrected fluxes. The DR method provides an efficient way to remove errors that result from a tilted sensor. The limitation involved with the DR method is the risk of over-rotation if a mean vertical velocity does indeed exist, which would be interpreted erroneously as a tilt. In this study, we carefully examined the 15-minute and 30-minute mean vertical velocities of all the collected data. Most of them are nonzero, which does not appear to be realistic. Therefore, the DR correction is applied to all the data. 


\section{RESULTS}

\subsection{Drag Coefficient}

In this study, $20 \mathrm{~Hz}$ data were collected at $5 \mathrm{~m}$ and $10 \mathrm{~m}$ (at the ENP and BBC sites), which allows the computation of higher order turbulent moments, such as turbulent fluxes and TKE, at two levels. Figure 4.1 compares frictional velocity $u_{*}$, kinematic sensible heat flux $\overline{w^{\prime} \theta^{\prime}}$, kinematic moisture flux $\overline{w^{\prime} q^{\prime}}$, and TKE of the data collected at the ENP site. The higher order turbulent moments at $10 \mathrm{~m}$ are slightly greater than those at $5 \mathrm{~m}$ at the high ends of the moments. The trend is more apparent for the frictional velocity, but overall the turbulent moments at two levels are fairly consistent. Similar results are found at the $\mathrm{BBC}$ site (not shown here). The consistency in turbulent moments at both measurement levels in these experiments supports the constant flux assumption of the SL and also gives increased confidence that the data collected by the tower provides a valuable sample for addressing issues regarding the SL vertical transport processes for overland conditions in coastal regions. 

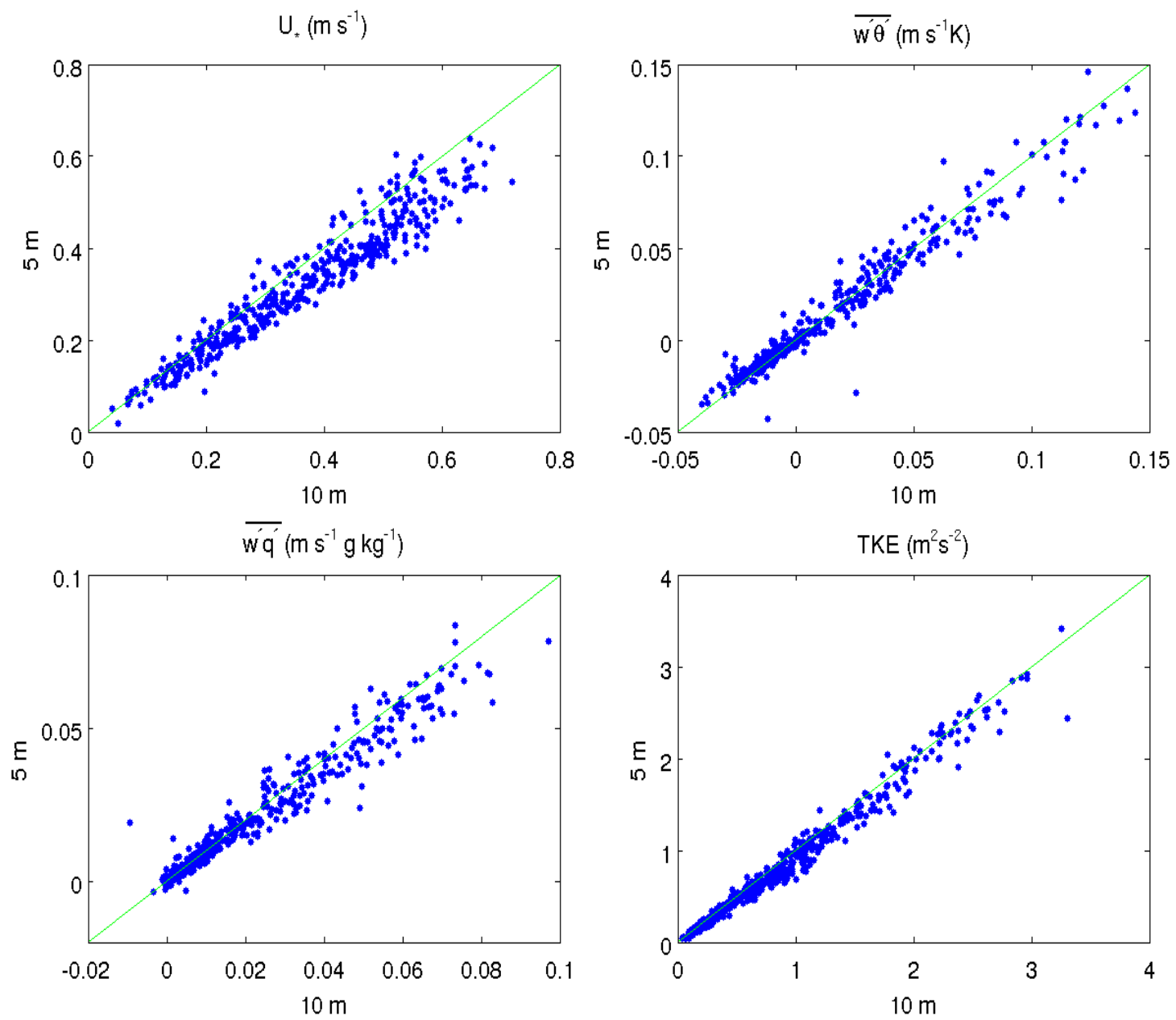

Figure 4.1 Plots of friction velocity, kinematic sensible heat flux, kinematic moisture flux, and TKE comparing the two measurement heights (5-m and 10-m) at the ENP site.

On the basis of the previously stated argument, the drag coefficient is computed using the data collected at $10 \mathrm{~m}$. Figure 4.2 shows the estimated $C_{D}$ values plotted against wind speed for all four sites. The data are scattered not only among different sites but also within the same site. Nonetheless, the drag coefficients derived from our measurements fall within the range of previous studies estimates shown in Figure 1.3. Moreover, despite the large spread in the data, an increasing trend of $C_{D}$ with a decrease in wind speed can still be seen. The causes of the large scattering of $C_{D}$ in the data are 
complex. Different surface roughness characteristics at different sites and different weather conditions including the harsh precipitating conditions at some times can all lead to varying estimates of $C_{D}$. Aside from the surface roughness and weather conditions, atmospheric stability is another important factor that can affect $C_{D}$. This effect is well described by the MOS framework (equations $1.12-1.14$ ).

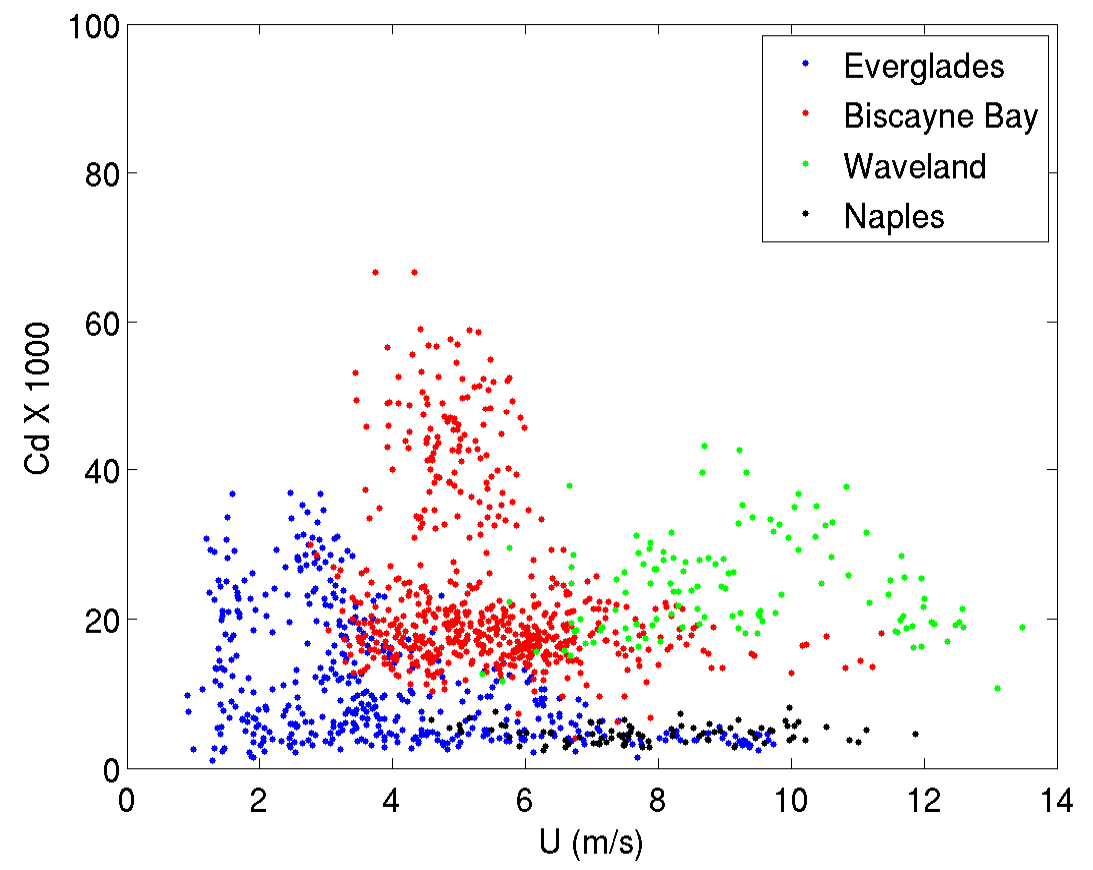

Figure 4.2 Plot of estimated values of $C_{D}$ against 10-m wind speed for all four sites.

The tower observations in this study allow the determination of atmospheric stability directly from the estimated heat and momentum fluxes. Figure 4.3 shows drag coefficient plotted against the dimensionless stability parameter $\varsigma=\frac{z}{L}$. Surprisingly, larger $C_{D}$ values occur in neutral conditions. This result contrasts MOS (equation 1.12), which predicts an increase in $C_{D}$ with a decrease in stability and larger $C_{D}$ values in 
unstable conditions. For a better illustration, Figure 4.4 shows $C_{D}$ against $\varsigma=\frac{z}{L}$ as predicted by MOS (equation 1.12). However, the results in this study appear to be consistent with previous observations. Patil (2006) analyzed data collected during the Land Surface Processes Experiment (LASPEX), which took place over a semi-arid area in India. The relationship between $C_{D}$ and $\varsigma=\frac{z}{L}$ that Patil (2006) found (Figure 4.5) shows a similar pattern to ours (Figure 4.3), i.e., $C_{D}$ is greater in near neutral conditions. Since both results (this study and Patil) are derived from two independent experiments, the results are credible. Currently, there is not an exact answer for the conflict between these two studies and the predictions from MOS. One possible explanation is that the estimation of $C_{D}$ from equation 1.12 (derived from MOS) does not explicitly depend on wind speed. However, observations show that $C_{D}$ has a strong dependence on wind speed even over land, particularly for low wind speeds. Another possible explanation is that there are other complicating factors that can substantially affect $C_{D}$ but have not been considered so far. This issue will be explored further in the next section. 


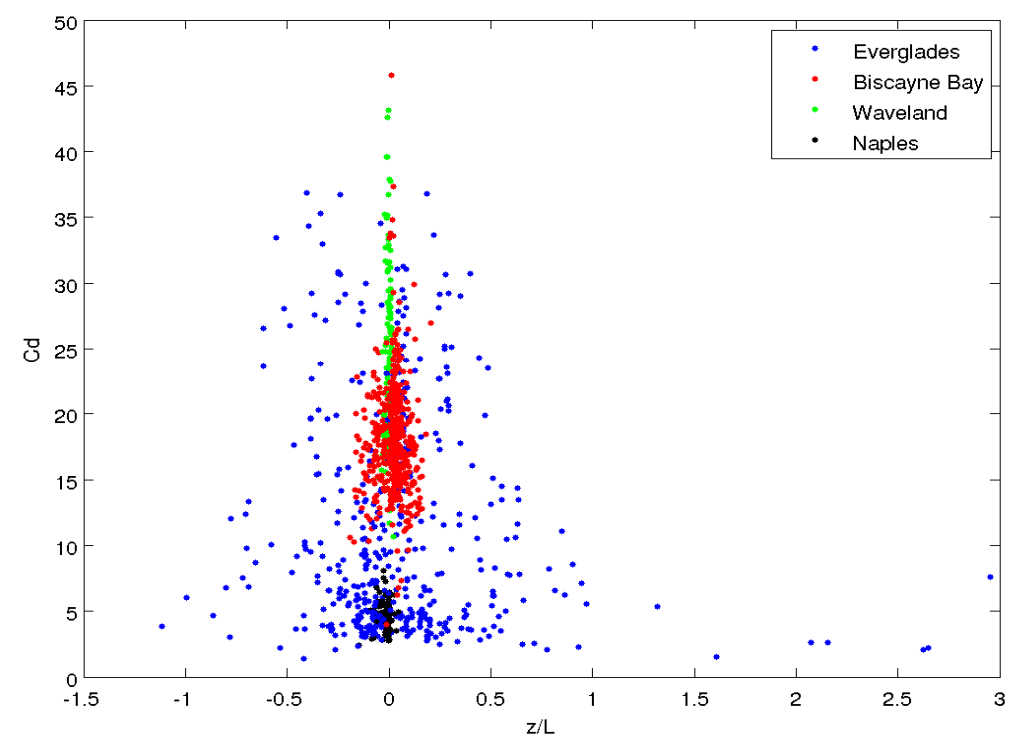

Figure 4.3 Plot of $C_{D}$ vs. the stability parameter $\varsigma=\frac{z}{L}$ for all four sites.

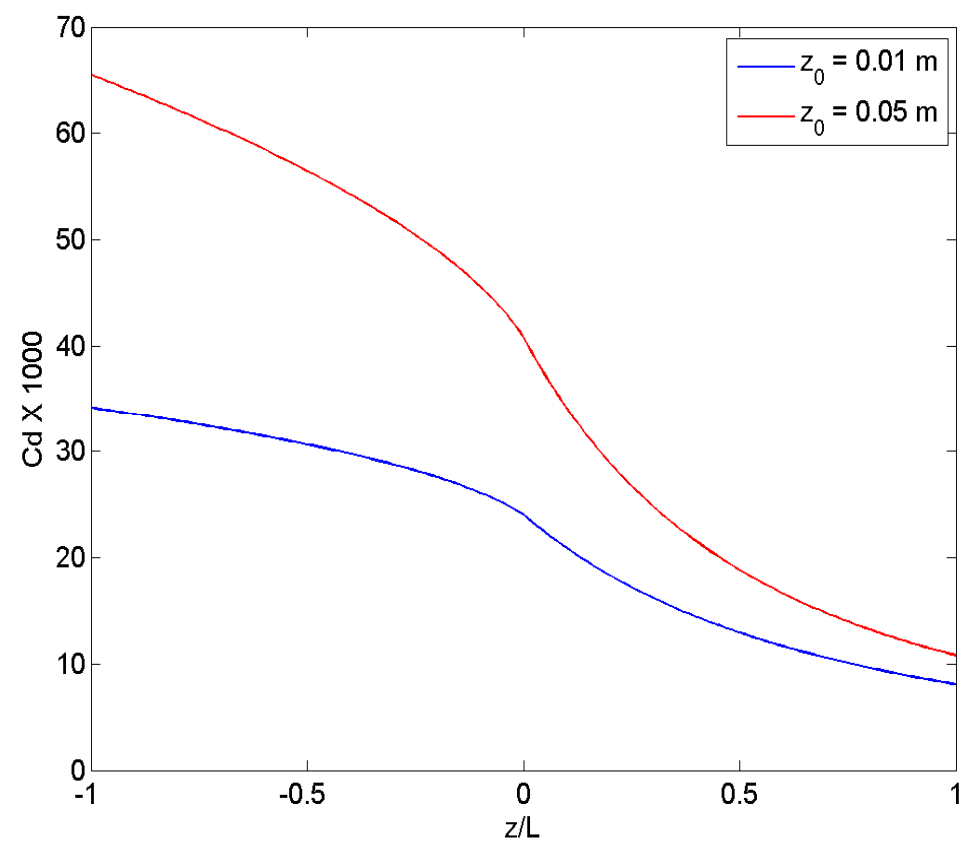

Figure 4.4 Comparison of $C_{D}$ vs. $\varsigma=\frac{z}{L}$ for two roughness lengths as predicted by MOS. 

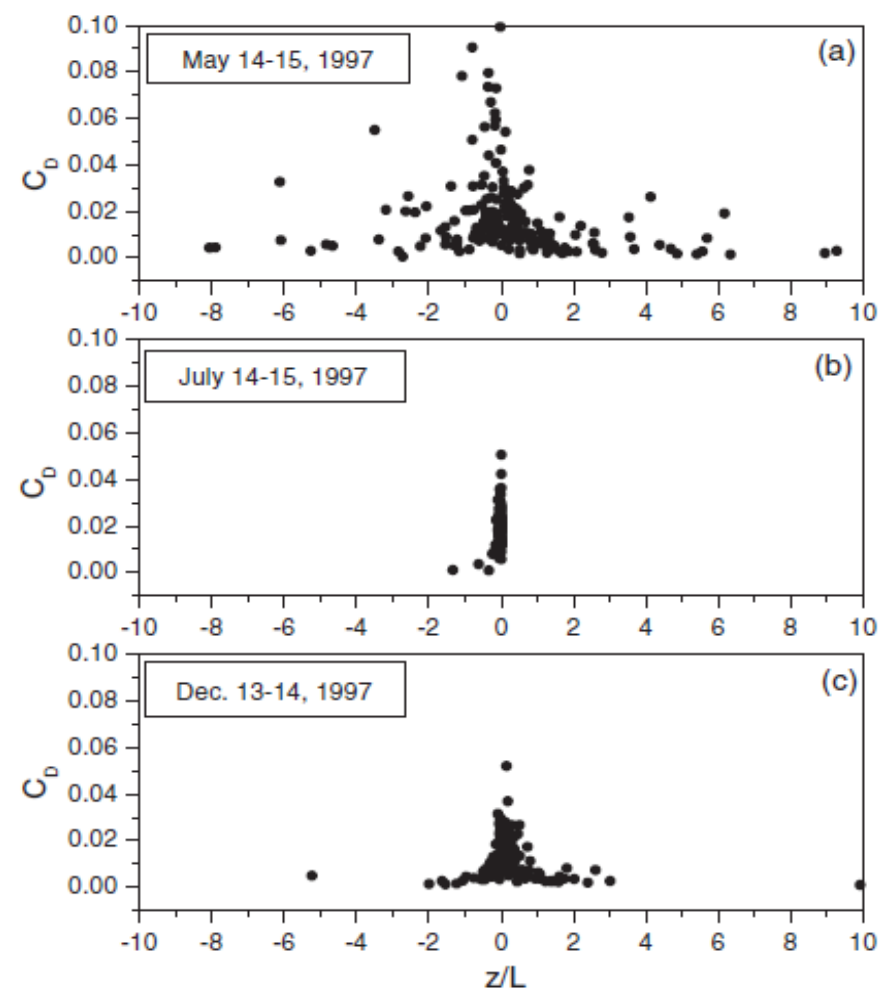

Figure 4.5 Plot adopted from Patil (2006) of $C_{D}$ vs. $\varsigma=\frac{z}{L}$.

To understand the large scatter of $C_{D}$ in this study, several analyses were completed by grouping $C_{D}$ into different categories on the basis of wind speed and stability, as well as normalizing $C_{D}$ using different variables such as TKE and vertical velocity variance. It turns out that there is a fairly good relationship among $C_{D}$, TKE, and wind speed. For example, Figure 4.6 shows $C_{D}$ plotted against TKE for all data collected at ENP, BBC, Waveland, and Naples. The data are scattered, however, as shown in the figure, the scatter can be explained well by wind speed. In each wind speed category, there exists a good relationship between $C_{D}$ and TKE. $C_{D}$ increases nearly linearly with 
TKE. Moreover, the slope of the line, i.e., the ratio of the change in $C_{D}$ to the change in TKE, increases with wind speed.

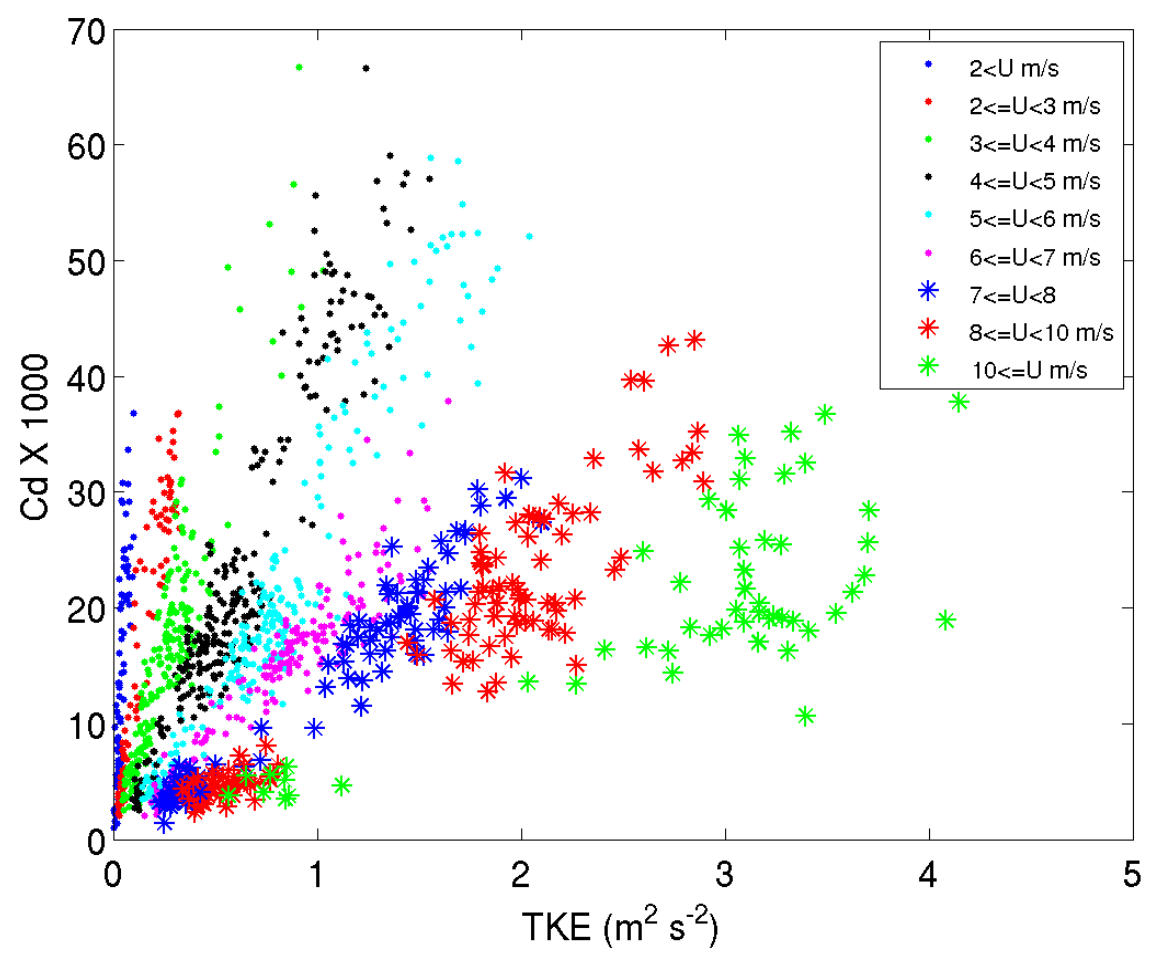

Figure 4.6 Plot of $C_{D}$ against TKE for all data collected at all four sites.

To further illustrate the strong linear relationship between $C_{D}$ and TKE as a function of wind speed, Figure 4.7 shows the ratio of $C_{D}$ to TKE plotted logarithmically against wind speed. All of the data from ENP, BBC, Waveland, and Naples fall nicely along a common line, and the slope of that line appears to be universal, inasmuch as the data was collected under various weather conditions. To further examine this relationship, Figure 4.7 shows the data collected during Hurricane Ivan (2004). The data from Ivan simply extends the line into higher wind speeds along the same slope. Since 
the data from Ivan was collected by a different instrument (propeller anemometer), and under different conditions, the findings for the ENP, BBC, Naples, and Waveland sites are consistent. Indeed, the correlation coefficient of all the data is approximately -0.997 .

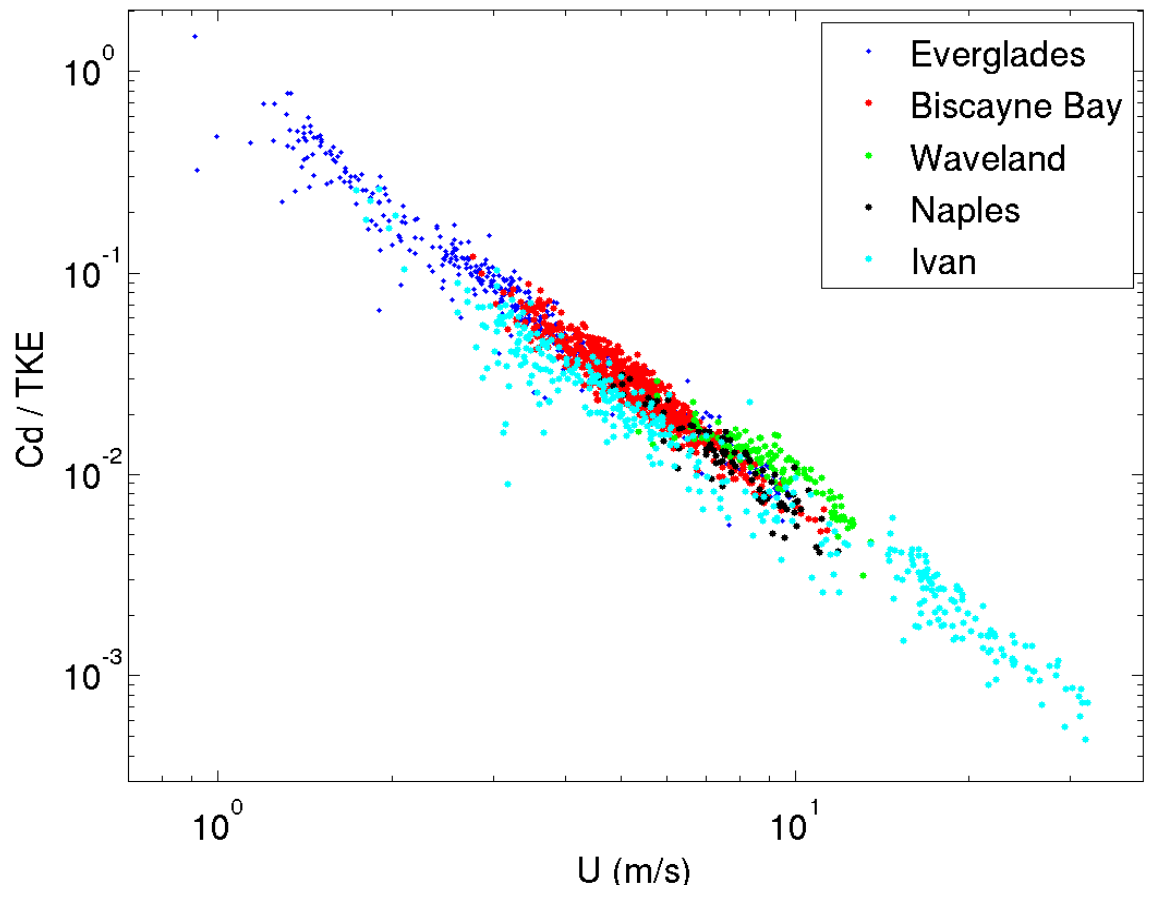

Figure 4.7 Logarithmic plot of the ratio of $C_{D}$ to TKE against 10-m wind speed for all four sites as well as data collected in Hurricane Ivan (2004).

Given that $C_{D}$ is a parameter that relates momentum fluxes to mean wind speed, the strong relationship among $C_{D}$, TKE, and wind speed suggests that momentum fluxes are well correlated to TKE. To confirm this, Figure 4.8 shows the total momentum flux (i.e., $u_{*}^{2}$ ) plotted against TKE. As expected, the two variables are well correlated for all the data. However, one note is that the linear relationship between $\ln \left(u_{*}^{2}\right)$ and $\ln (\mathrm{TKE})$ does not appear to hold for small $u_{*}$. The best fit curve (red) deviates from the linear fit 
line (blue) toward larger TKE suggesting that it may not be sufficient to consider only stability or wind speed when parameterizing $C_{D}$. As shown here, an appropriate determination of $C_{D}$ needs to take into account turbulent intensity as well.

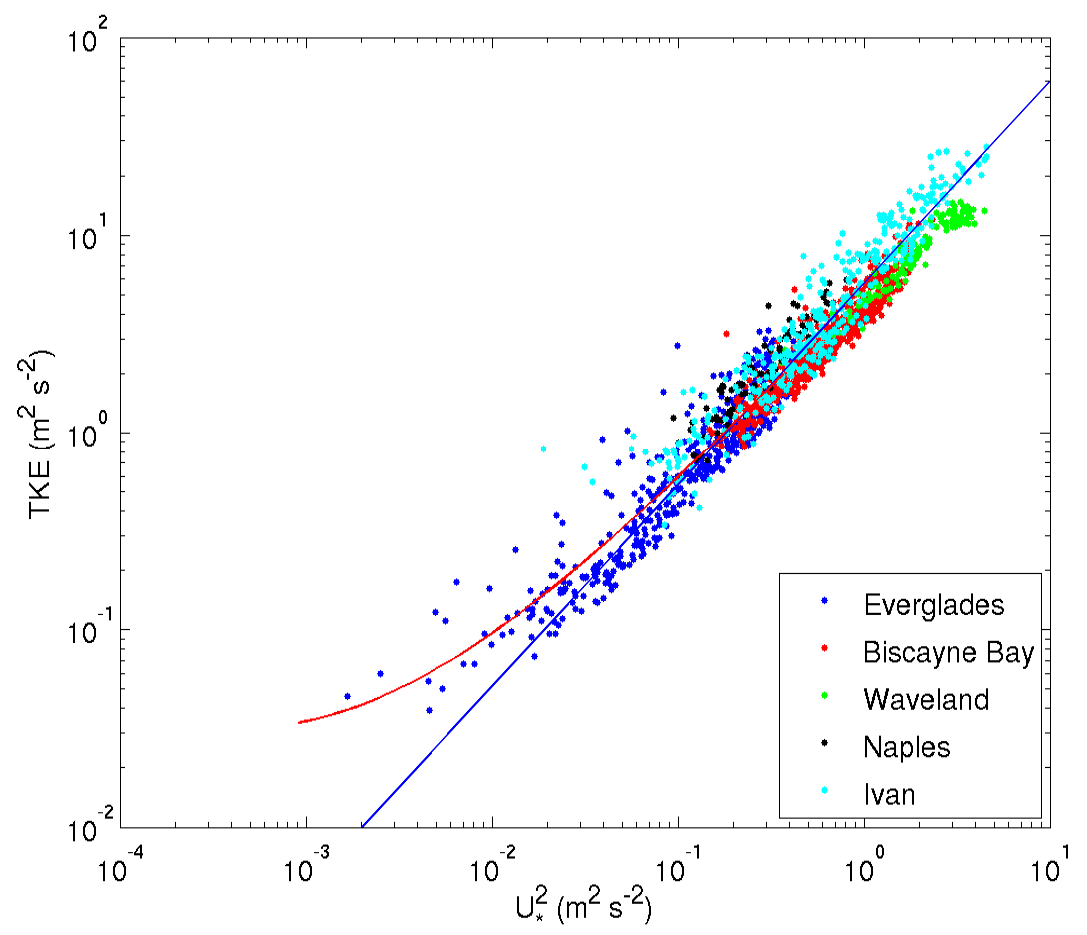

Figure 4.8 Logarithmic plot of the total momentum flux against TKE for all four sites, including Hurricane Ivan (2004).

\subsection{A New Perspective on Bulk Exchange Coefficients}

The previous section demonstrated that there is a strong correlation between momentum fluxes and TKE. This result suggests that the parameterization of momentum fluxes may be explored by analyzing the TKE budget. Assuming horizontal homogeneity, and aligning the $x$-axis along the mean wind direction, the TKE budget may be written as: 


$$
\frac{\partial e}{\partial t}=-\overline{u^{\prime} w^{\prime}} \frac{\partial \bar{u}}{\partial z}+\frac{g}{\theta_{0}} \overline{w^{\prime} \theta^{\prime}}-\frac{\partial \overline{w^{\prime} e}}{\partial z}-\frac{1}{\rho_{0}} \frac{\partial \overline{w^{\prime} p^{\prime}}}{\partial z}-\varepsilon_{e}
$$

where $e$ represents TKE, $p$ is pressure, and $g$ is gravity. $\theta_{0}$ and $\rho_{0}$ are ambient potential temperature and air density, respectively. The term on the left-hand side (LHS) is the local storage term. The first, second, third, fourth, and fifth terms on the right-hand side (RHS) of equation 4.1 are the TKE shear production, buoyancy production, vertical transport, pressure correlation, and dissipation, respectively.

Furthermore, $\overline{w^{\prime} e}$ and $\overline{w^{\prime} p^{\prime}}$ in equation 4.1 are the vertical fluxes of TKE and pressure work. Note that the SL is also known as the constant flux layer. Thus, the third and fourth terms, i.e., vertical transport term and pressure correlation term, on the RHS of

equation 4.1 are negligible. For a steady state $\mathrm{SL}, \frac{\partial e}{\partial t}=0$ is also true. With these assumptions, the TKE budget equation can be simplified. Next we will consider neutral and non-neutral conditions separately.

\subsubsection{Neutral Condition}

Under neutral atmospheric conditions (i.e., $\overline{w^{\prime} \theta^{\prime}}=0$ ), equation 4.1 becomes:

$$
-\overline{u^{\prime} w^{\prime}} \frac{\partial \bar{u}}{\partial z}=\varepsilon_{e}
$$

Equation 4.2 simply states a balance between TKE shear production (LHS) and TKE dissipation (RHS). In higher order turbulent closure models (e.g., Mellor and Yamada 1974; Zeman, 1981; Deardorff 1973), the TKE dissipation is often parameterized as:

$$
\varepsilon_{e}=\frac{e^{3 / 2}}{\Lambda}
$$


where, $\Lambda$ is an empirical dissipation length scale. Subsequently, the momentum fluxes may be written as:

$$
-\overline{u^{\prime} w^{\prime}}=u_{*}^{2} .
$$

In the previous section, it was shown that there is a strong log linear relationship between TKE and frictional velocity, which may be represented as:

$$
u_{*}^{2}=c_{1} e,
$$

where, $c_{l}$ is an empirical coefficient. Combining equations $4.2-4.5$, we obtain:

$$
\frac{c_{1}^{3 / 2} \Lambda}{u_{*}} \frac{\partial \bar{u}}{\partial z}=1 .
$$

In higher order turbulent closure (e.g., Mellor and Yamada 1974; Zeman, 1981; Deardorff 1973), the empirical dissipation length scale $\Lambda$ in the SL is often considered as a function of height:

$$
\Lambda=c_{2} z,
$$

where $c_{2}$ is an empirical coefficient. Then, equation 4.6 may be written in another format as:

$$
\frac{c_{1}^{3 / 2} c_{2} z}{u_{*}} \frac{\partial \bar{u}}{\partial z}=1
$$

\subsubsection{Case 1}

Assuming the empirical coefficients $c_{1}$ and $c_{2}$ are constants the Von-Karman constant can be defined as:

$$
\kappa=c_{1}^{3 / 2} c_{2} .
$$


Then, equation 4.8 simply becomes:

$$
\frac{\kappa z}{u_{*}} \frac{\partial \bar{u}}{\partial z}=1 .
$$

What is obtained here is the famous MOS relationship in neutral conditions. Historically, the MOS relationship was derived from dimensional analysis, or Buckingham $\pi$ theorem. Here, it is shown that the famous MOS relationship in neutral atmospheric conditions can be obtained from the TKE budget equation with the appropriate assumptions.

\subsubsection{Case 2}

In the previous analysis, it was shown that $u_{*}$ and TKE do not have a linear relationship at low wind speeds. Hence, it is suspected that the coefficient $\kappa=c_{1}^{3 / 2} c_{2}$ (equation 4.9) may not be a constant as previously assumed. Instead, equation 4.9 may depend on wind speed. The empirical coefficient $c_{1}$ can be estimated from values of $u_{*}$ and TKE calculated in this study (Figure 4.8). To estimate $c_{2}$, one needs to know the TKE dissipation rate $\varepsilon_{e}$ (equation 4.3), which may be calculated directly from Kolmogorov's energy spectrum law. Kolmogorov first showed that in the inertial subrange, in an equilibrium state, the energy density per unit wave number depends only on the wave number and the rate of energy dissipation, $\varepsilon_{e}$. Dimensional analysis yields:

$$
S(v)=\alpha \varepsilon_{e}^{2 / 3} v^{-5 / 3}
$$

where $v$ is the angular wave number, $S(v)$ is the energy density per unit wave number in the inertial sub-range, and $\alpha$ is the universal Kolmogorov constant. Equation 4.11 is also 
known as Kolmogorov's 5/3 power law. Converting equation 4.11 from wave number domain to frequency domain, one obtains:

$$
\varepsilon_{e}=\alpha^{-3 / 2} \frac{2 \pi f}{U}[f S(f)]^{3 / 2}
$$

where the relationship $v=\frac{2 \pi f}{U}$ is used. $U$ is the mean wind speed, and $S(f)$ is the energy density per unit frequency.

Theoretically, the energy density of the three wind components $u$, $v$, and $w$ should be the same in the inertial sub-range. In this study, we have carefully examined the turbulence spectra of all the data. As an example, Figure 4.9 shows the energy density power spectra of $u, v$, and $w$ of an arbitrary spectral lag (15 min) from the ENP data. Several features are shown in the figure. First, in the inertial sub-range, the spectra of $u$, $v$, and $w$ all follow the $-5 / 3$ power law nicely, furthering illustrating the quality of this study's data observations. Second, the magnitudes of spectra of the different wind components are nearly the same in the inertial sub-range, which is consistent with the theoretical derivation. This suggests that the energy dissipation rate can be estimated using both horizontal and vertical wind components. Third, for the energy containing eddies, the magnitude of $w$ spectra is substantially smaller than that of horizontal ( $u$ and v) wind spectra. This result is consistent with previous studies (e.g., Kaimal et al. 1972; 1976; and Busch 1973).

On the basis of the spectra obtained in this study, a frequency band of $0.6-5 \mathrm{~Hz}$ (indicated by the vertical lines in Figure 4.9) is chosen as the inertial sub-range when estimating $\varepsilon_{e}$. As an example, Figure 4.10 shows the estimated $\varepsilon_{e}$ at each frequency in 
the inertial sub-range. Although the spread of the data is fairly large, there is no apparent trend. Thus, the averaged $\varepsilon_{e}$ over the frequency band is considered as the mean $\varepsilon_{e}$ of a spectrum lag (15min). Once $\varepsilon_{e}$ is determined, the coefficient $c_{2}$ can be estimated using equation 4.3 and equation 4.7 .

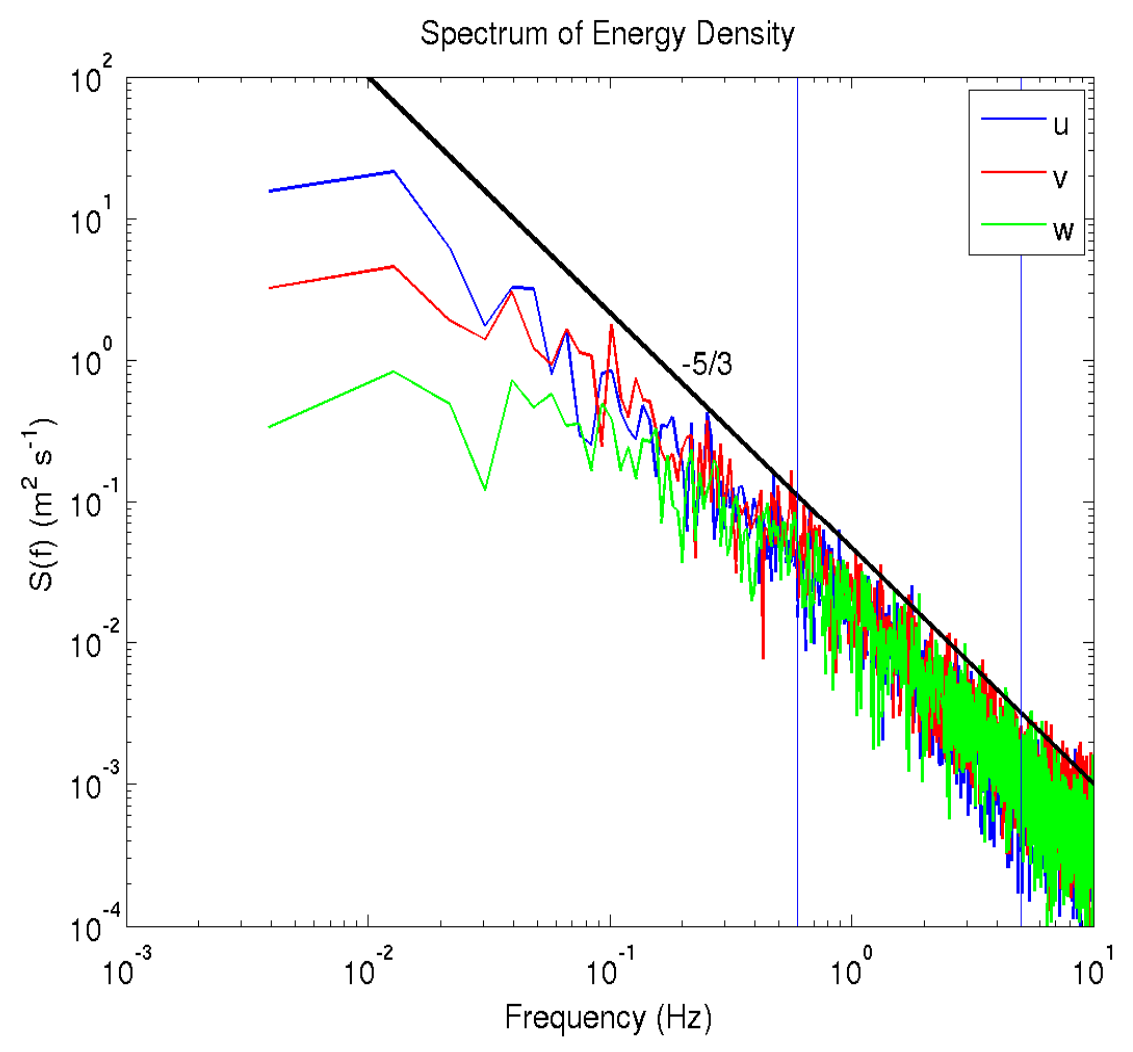

Figure 4.9 Plot of the energy density power spectra of $u, v$, and $w$ for an arbitrary 15 minute spectral lag from the ENP data. 


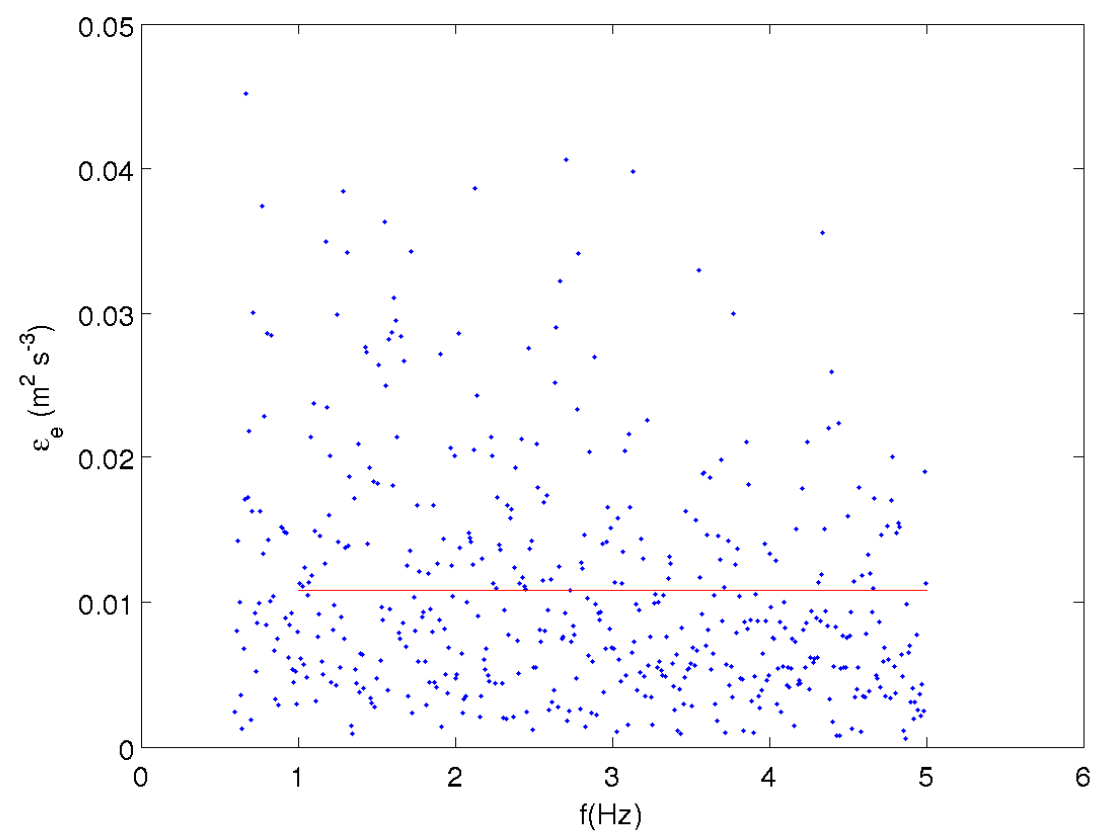

Figure 4.10 Plot of the estimated energy dissipation rate $\left(\varepsilon_{e}\right)$ at each frequency in the inertial sub-range.

Figure 4.11 shows the estimated coefficient $\kappa=c_{1}^{3 / 2} c_{2}$ plotted against wind speed. Note that all data shown in the figure are from near neutral conditions $\left(0.1<\left(\varsigma=\frac{z}{L}\right)<0.1\right)$ for the ENP, BBC, Waveland, and Naples sites. For Ivan, there are no temperature and moisture measurements available, thus a direct estimate of stability is impossible. In that case, the stability is estimated indirectly from the wind measurements at $5 \mathrm{~m}$ and $10 \mathrm{~m}$ by solving the MOS iteratively. Figure 4.11 clearly shows that the coefficient $\kappa=c_{1}^{3 / 2} c_{2}$ is not a constant but instead increases with wind speed, and substantially increases at low wind speeds. The best fit curve shows the best estimates of $\kappa=c_{1}^{3 / 2} c_{2}$, which can be represented as: 


$$
\kappa=c_{1}^{3 / 2} c_{2}=\kappa^{*}\left[1+c_{3} * \exp \left(-c_{4} \bar{u}\right)\right], \quad \kappa=0.35, \quad c_{3}=5.0, \quad c_{4}=0.5
$$

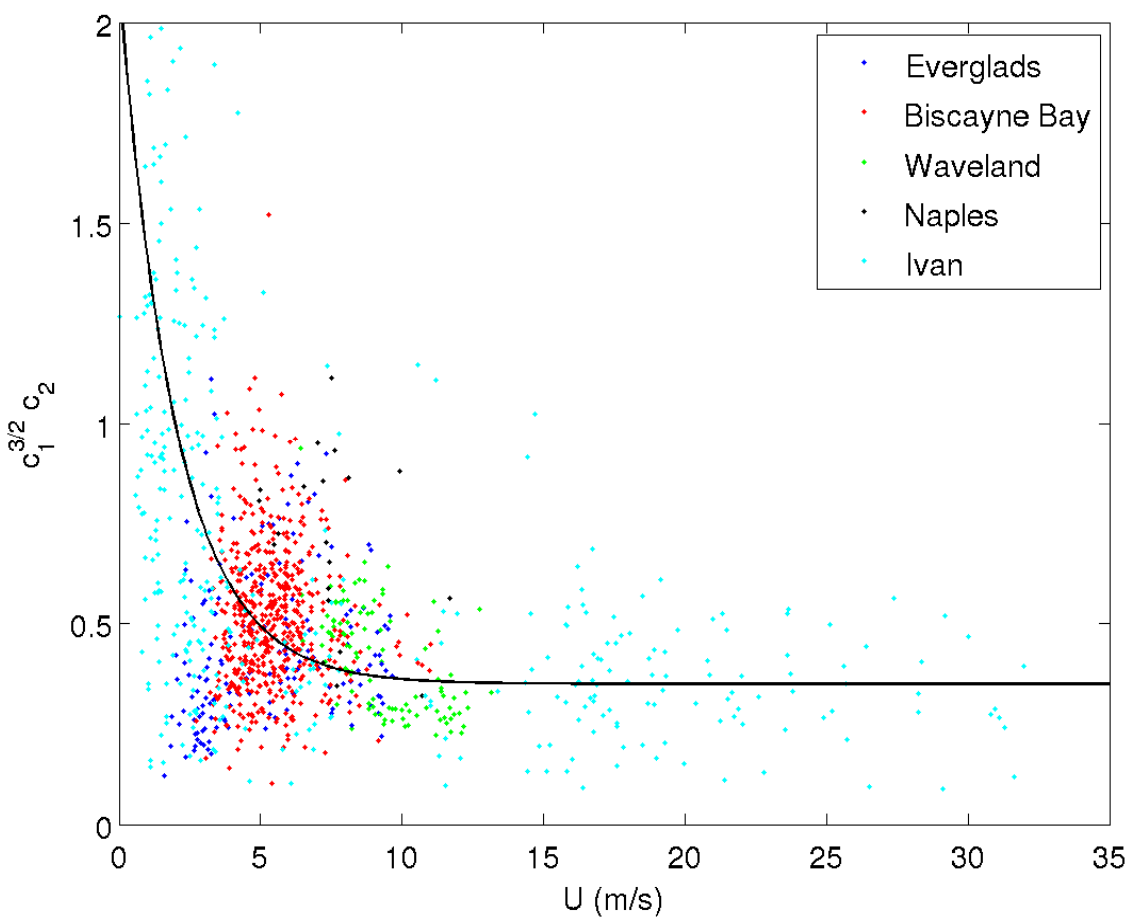

Figure 4.11 Plot of the estimated coefficient $\kappa=c_{1}^{3 / 2} c_{2}$ against $10-\mathrm{m}$ wind speed for all data from all four sites including Hurricane Ivan (2004).

With equation 4.13 , equation 4.8 becomes:

$$
\frac{\kappa z\left(1+c_{3} e^{-c_{4} \bar{u}}\right)}{u_{*}} \frac{\partial \bar{u}}{\partial z}=1
$$

Then, it can be shown that the drag coefficient in neutral conditions can be written as:

$$
C_{D N}=\frac{\kappa^{2}}{\left[\ln \left(z / z_{0}\right)\right]^{2}}\left[1+\frac{c_{3}}{c_{4} U_{10}}\left(1-e^{-c_{4} U_{10}}\right)\right]^{2} .
$$


Figure 4.12 shows the values of $C_{D N}$ computed from MOS (equation 1.12) and from equation 4.15. The revised $C_{D N}$ adequately explains the observed, variable pattern of $C_{D N}$ (Figure 3).

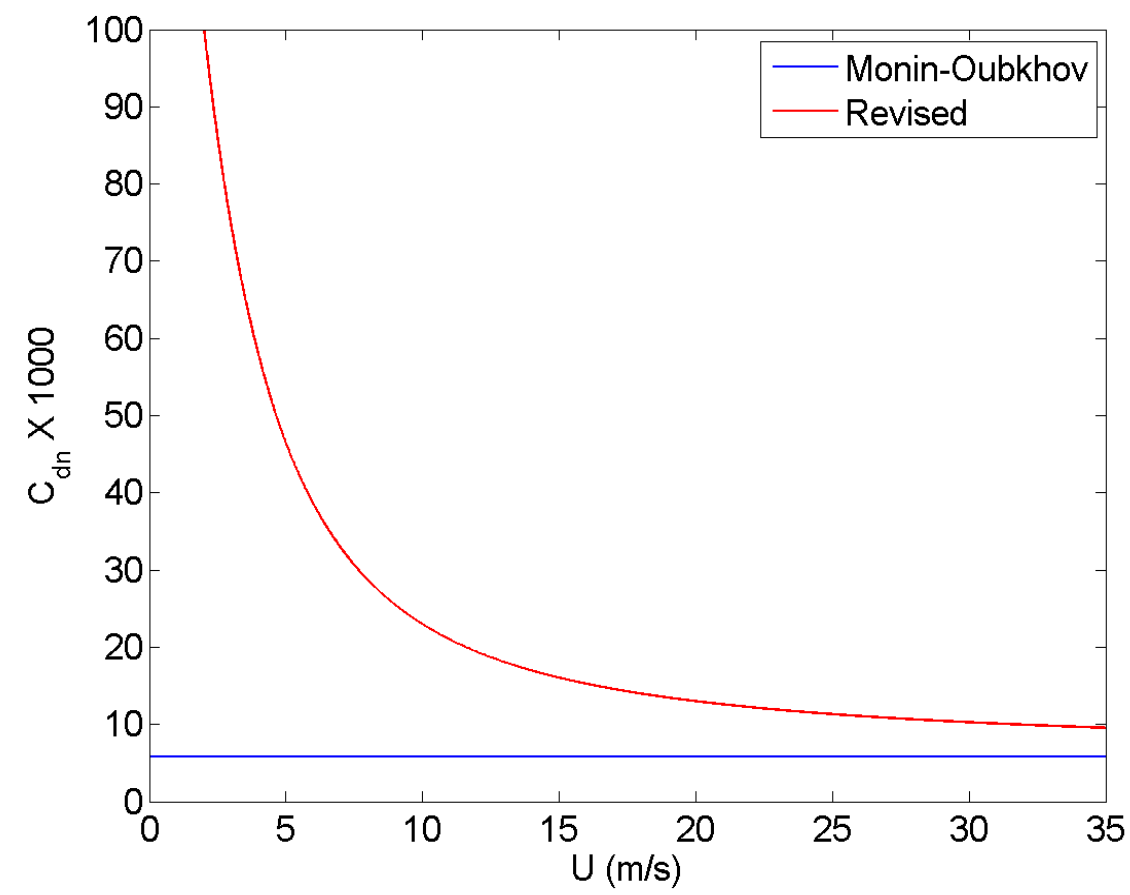

Figure 4.12 Plot of $C_{D N}$ values computed from MOS (blue) and from equation 4.15 (red) against 10-m wind speed.

\subsubsection{Non-neutral Condition}

In the non-neutral condition, the TKE buoyancy production needs to be considered. The TKE budget equation then becomes:

$$
-\overline{u^{\prime} w^{\prime}} \frac{\partial \bar{u}}{\partial z}+\frac{g}{\theta_{0}} \overline{w^{\prime} \theta^{\prime}}=\varepsilon_{e} .
$$

Normalizing equation 4.16 by the shear production term yields: 


$$
1+\frac{\frac{g}{\theta_{0}} \overline{w^{\prime} \theta^{\prime}}}{-\overline{u^{\prime} w^{\prime}} \frac{\partial \bar{u}}{\partial z}}=\frac{\varepsilon_{e}}{-\overline{u^{\prime} w^{\prime}} \frac{\partial \bar{u}}{\partial z}}, \text { or } 1-R_{f}=\frac{\varepsilon_{e}}{-\overline{u^{\prime} w^{\prime}} \frac{\partial \bar{u}}{\partial z}}
$$

where, $R_{f}=\frac{g}{\theta_{0}} \overline{w^{\prime} \theta^{\prime}} / \overline{u^{\prime} w^{\prime}} \frac{\partial \bar{u}}{\partial z}$ is defined as the flux Richardson number. Further applying equations $4.3-4.5$, and using the definition of the Monin-Obukhov length $\left(L=\frac{-u_{*}^{3} \bar{\theta}}{k g \overline{w^{\prime} \theta} \theta^{\prime}}\right)$, equation 4.17 can be rewritten as:

$$
\frac{c_{1}^{3 / 2} c_{2} z}{u_{*}} \frac{\partial \bar{u}}{\partial z}=\frac{1}{1-\varsigma}
$$

Comparing equation 4.18 with MOS (equation 1.4), it is easy to see that the two equations have the same format. The term on the left side of the equation is the same as that found in the neutral condition. The effect of stability is represented by the term on the RHS of the equation. One advantage of this parameterization framework is that the effect of stability is directly determined from the TKE budget itself. This contrasts MOS, in which the stability function $\left(\phi_{m}[\varsigma]\right)$ has to be determined empirically. However, one note is that since the dissipation rate $\varepsilon_{e}$ has to be positive, equation 4.18 is valid only for $R_{f}<1$ or $\varsigma=\frac{z}{L}<1$. This argument is logical since under stable conditions, the TKE shear production term must be greater than the buoyancy suppression term to maintain an equilibrium turbulent state; otherwise, turbulence will eventually die away as a result of the larger turbulent buoyancy suppression. MOS (equation 1.4) does not have a limit for stability simply because the stability function is determined empirically. Nevertheless, the effect of stability from this study's derivation is consistent with the empirical stability 
effect from MOS. For example, in unstable conditions $(\varsigma<0)$, the stability function $\frac{1}{1-\varsigma}<1$ is consistent with the MOS stability function $\phi_{m}(\varsigma)<1$. In stable conditions $(\varsigma>0)$, this study's stability function $\frac{1}{1-\varsigma}>1$ is consistent with the MOS stability function $\phi_{m}(\varsigma)>1$

Integrating equation 4.18 , one can determine the drag coefficient:

$$
C_{D}=\frac{\kappa^{2}}{\left[\ln \left(z / z_{0}\right)-\ln (1-\varsigma)\right]^{2}}\left[1+\frac{c_{3}}{c_{4} U_{10}}\left(1-e^{-c_{4} U_{10}}\right)\right]^{2} .
$$

Figure 4.13 shows $C_{D}$ as a function of stability and wind speed. Apparently, for particular wind and stability distributions, it is possible to produce a $C_{D}$ distribution pattern similar to what is shown in Figures 4.3 and 4.5. To illustrate this concept, $C_{D}$ is re-plotted against stability for different wind speed ranges in Figure 4.14. Since there are different surface roughness characteristics at each of the four collection sites, this can lead to different $C_{D}$ according to equations $1.12-1.14$. It is clear that the large spread of $C_{D}$ basically reflects the complicated effects of stability and wind speed on $C_{D}$. 


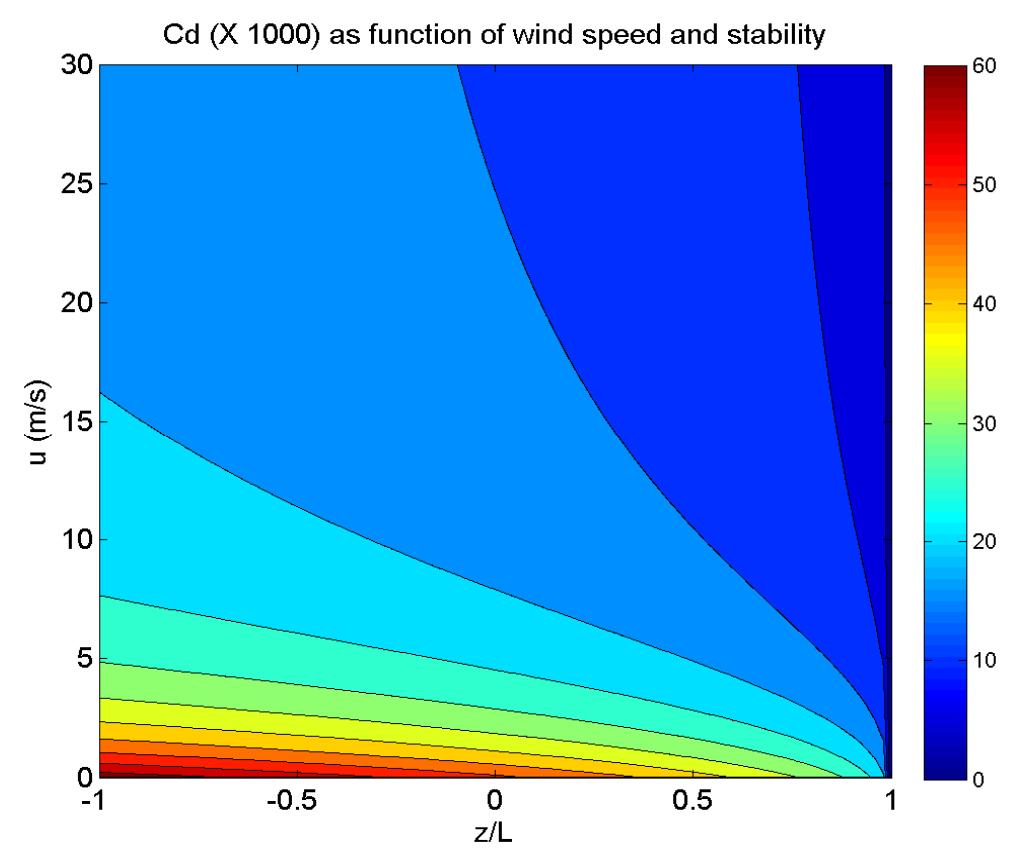

Figure 4.13 Plot of $C_{D}$ as a function of both 10-m wind speed and the stability parameter $\varsigma=\frac{z}{L}$.

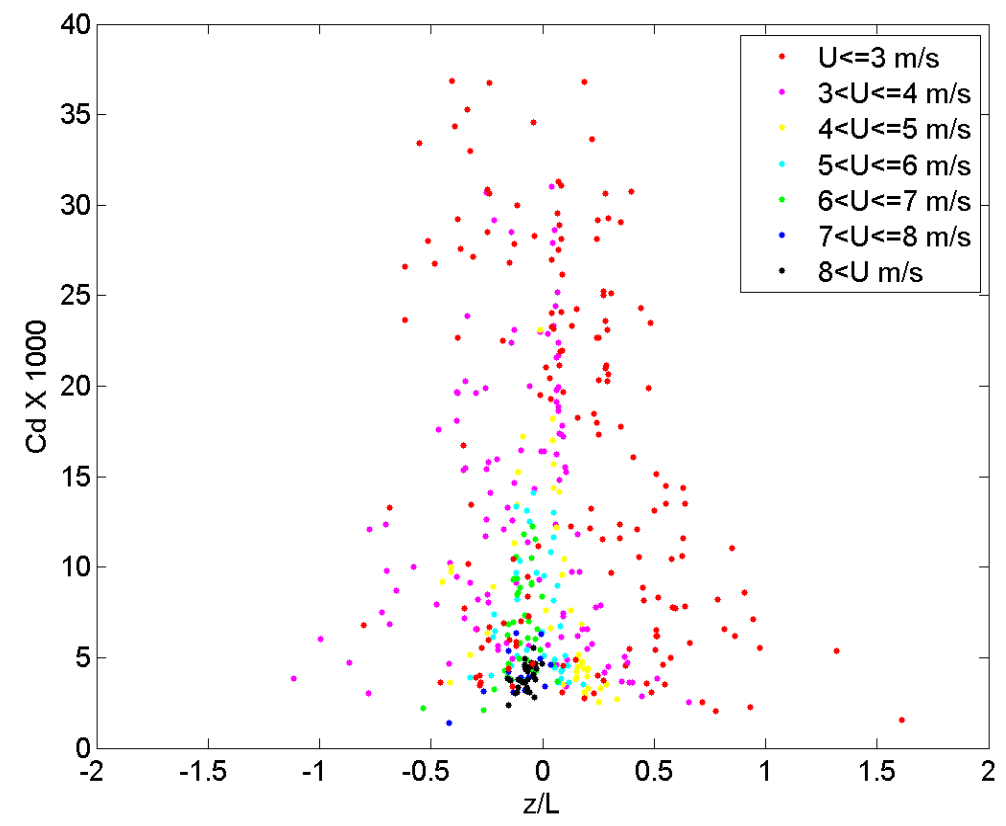

Figure 4.14 Plot of $C_{D}$ against $\varsigma=\frac{z}{L}$ for different wind speed ranges. 


\subsection{Exchange Coefficients for Heat and Moisture}

As indicated by equations $1.2-1.3$, an accurate determination of $C_{H}$ and $C_{Q}$ requires temperature and moisture observations at the ground surface. The tower deployments at Naples and Waveland do not contain surface temperature measurements, therefore the discussion on $C_{H}$ and $C_{Q}$ in this section will focus on the observations at the ENP and BBC sites. Compared with $C_{D}$, estimating $C_{H}$ and $C_{Q}$ requires a ground temperature and moisture measurement, introducing an extra component of uncertainty. In the bulk transfer parameterization, the ground temperature and moisture are also known as the "skin" temperature and moisture of the ground surface, which often refer to the values $\sim 1 \mathrm{~mm}$ above the soil or sea surface. Unfortunately, obtaining high resolution temperature and moisture measurements at $1 \mathrm{~mm}$ is difficult. The reason is the existence of a large vertical gradient in temperature close to the ground surface, particularly on sunny days. In addition, radiation and precipitation can also substantially affect the "skin" temperature measurement. Thus, one should bear in mind that there is an inherent uncertainty in estimating $C_{H}$ and $C_{Q}$ resulting from surface measurements when interpreting the results.

The tower deployments at the ENP and BBC sites successfully collected observations for several days, which allow the examination of the diurnal variations of exchange coefficients in coastal regions. Figures 4.15 and 4.16 show the time series of surface wind speeds, buoyancy fluxes, and exchange coefficients obtained at the ENP and BBC sites. As expected, the surface buoyancy fluxes show a clear diurnal cycle. 
Although it is not as clear as the buoyancy fluxes, $C_{H}$ at both sites shows a similar diurnal variation that follows the buoyancy flux profile. However, no diurnal variations are seen in $C_{Q}$ and $C_{D}$.

Interestingly, wind speeds at the ENP site also show a somewhat diurnal pattern that follows the variation of the buoyancy fluxes. No such diurnal variations of wind are present at the $\mathrm{BBC}$ site. This may be because the observations at the $\mathrm{BBC}$ were influenced by the passage of Hurricane Sandy during the data collection. However one note is that the diurnal variation of winds at the ENP site does not appear to have an effect on the variations of exchange coefficients. There are variations shown in $C_{D}$, but they do not simply follow a typical diurnal cycle.

As shown previously, multiple factors, such as wind speed, stability, and turbulent intensity can all affect the value of $C_{D}$. The values of $C_{Q}$ are generally small compared with $C_{D}$ and $C_{H}$, but there are occasional periods of large $C_{Q}$ occurring in the time series. The cause for very large $C_{Q}$ (and $C_{H}$ as well) is complicated. One cause is that the estimate of $C_{Q}$ and $C_{H}$ involves a singularity measurement. When $\left(\bar{\theta}-\theta_{G}\right)$ or $\left(\bar{q}-q_{G}\right)$ approaches zero (in equations $\left.1.2-1.3\right)$, it can lead to extremely large values of $C_{Q}$ and $C_{H}$. Thus, the occasional large values of $C_{Q}$ and $C_{H}$ may not be realistic. 
Everglades site
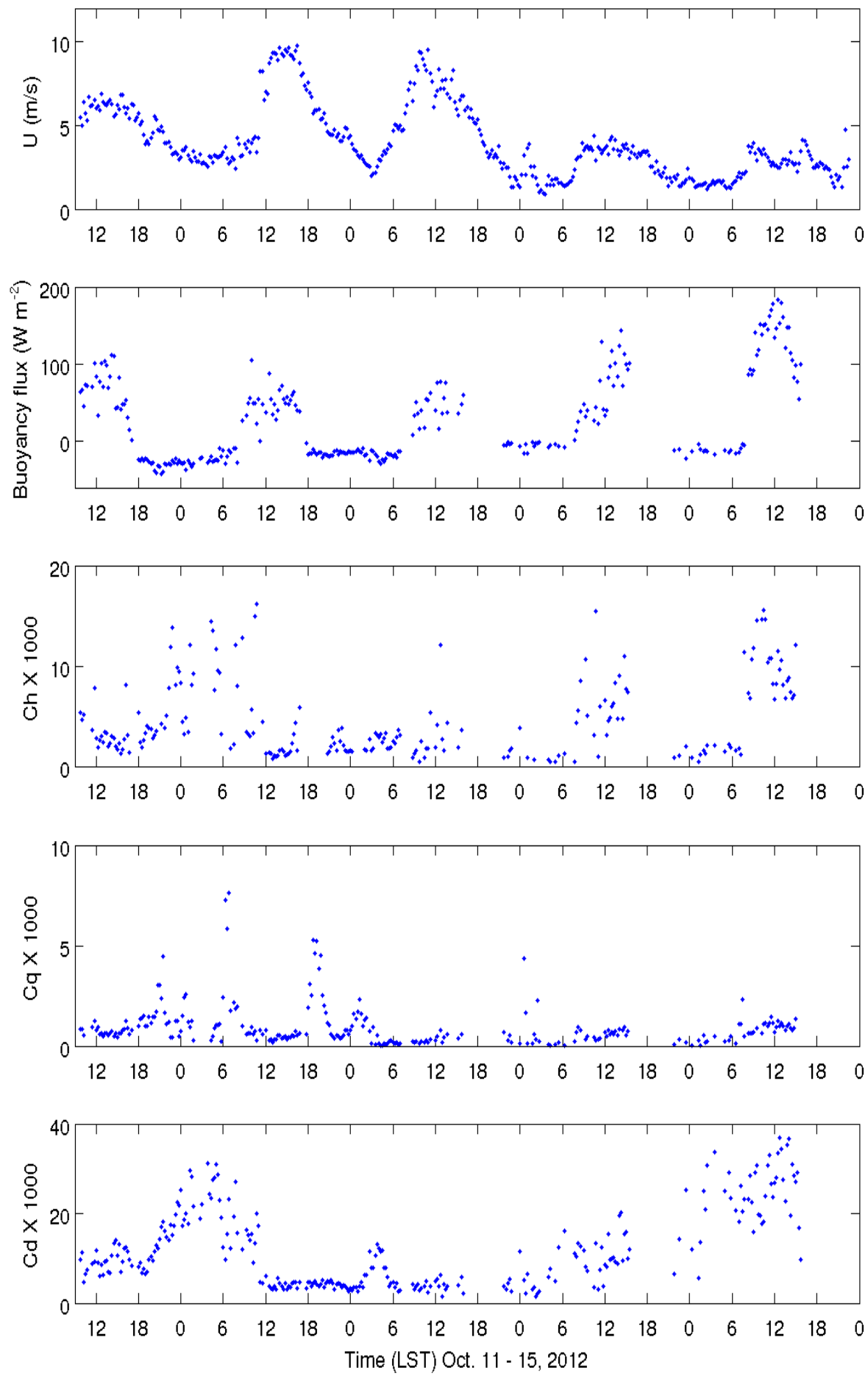

Figure 4.15 Time series of wind speed, buoyancy flux, and exchange coefficients for the ENP site. 

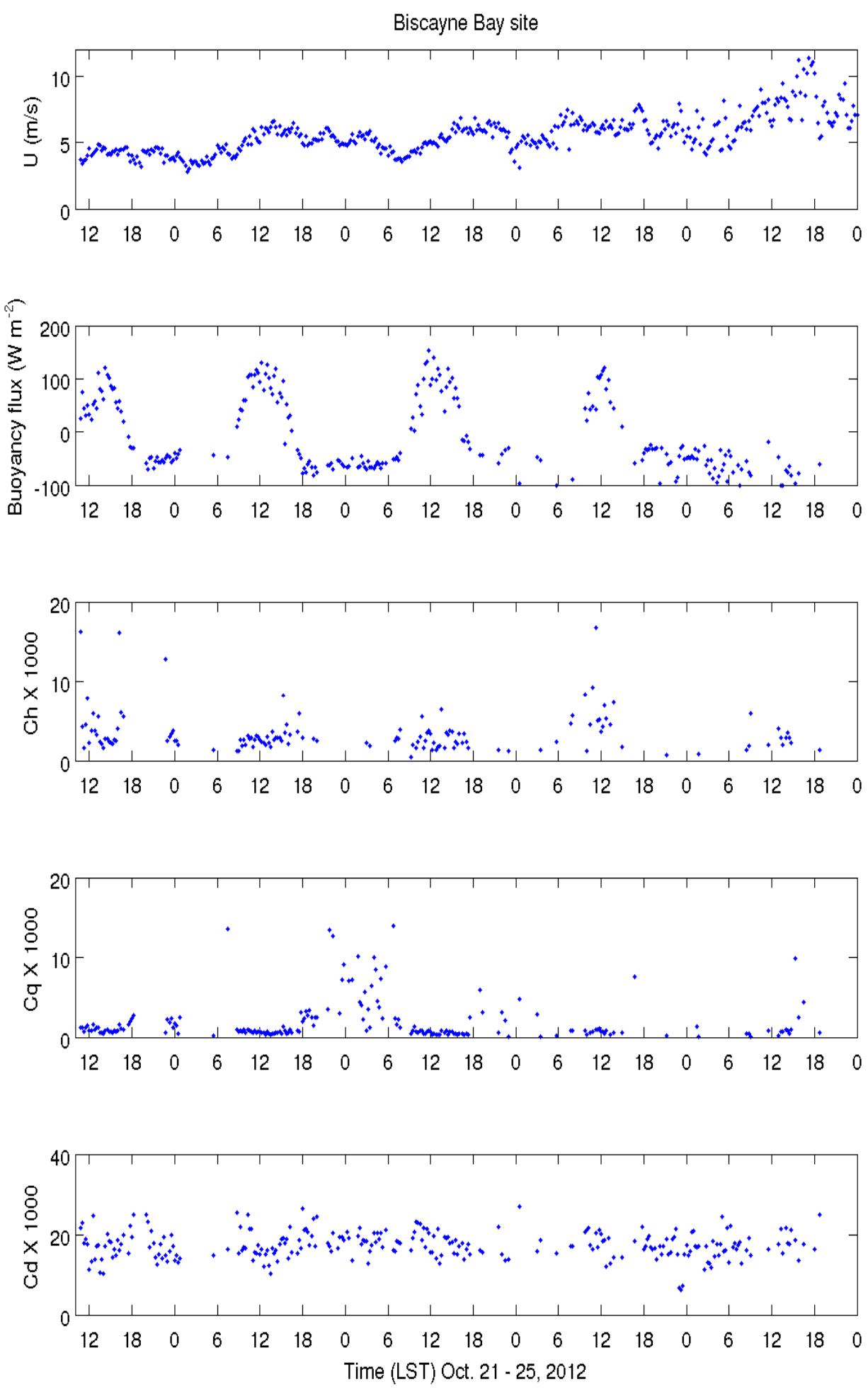

Figure 4.16 Time series of wind speed, buoyancy flux, and exchange coefficients for the $\mathrm{BBC}$ site. 
For comparison, similar to the analysis of $C_{D}, C_{H}$ and $C_{Q}$ are plotted against the wind speed (Figure 4.17). Unlike $C_{D}$, which shows a strong dependence on wind speed in the low wind speed regime, no apparent trend of $C_{H}$ and $C_{Q}$ varying with wind speed is observed for both the ENP and BBC sites. Rather, the data are fairly scattered. Nonetheless, the mean magnitude of $C_{H}$ and $C_{Q}$ obtained from the two sites are comparable, and are close to that of previous studies (e.g., Grachev et al. 2011; Rao 2004). This result indicates that wind speed may not be a critical parameter that affects the value of $C_{H}$ and $C_{Q}$.
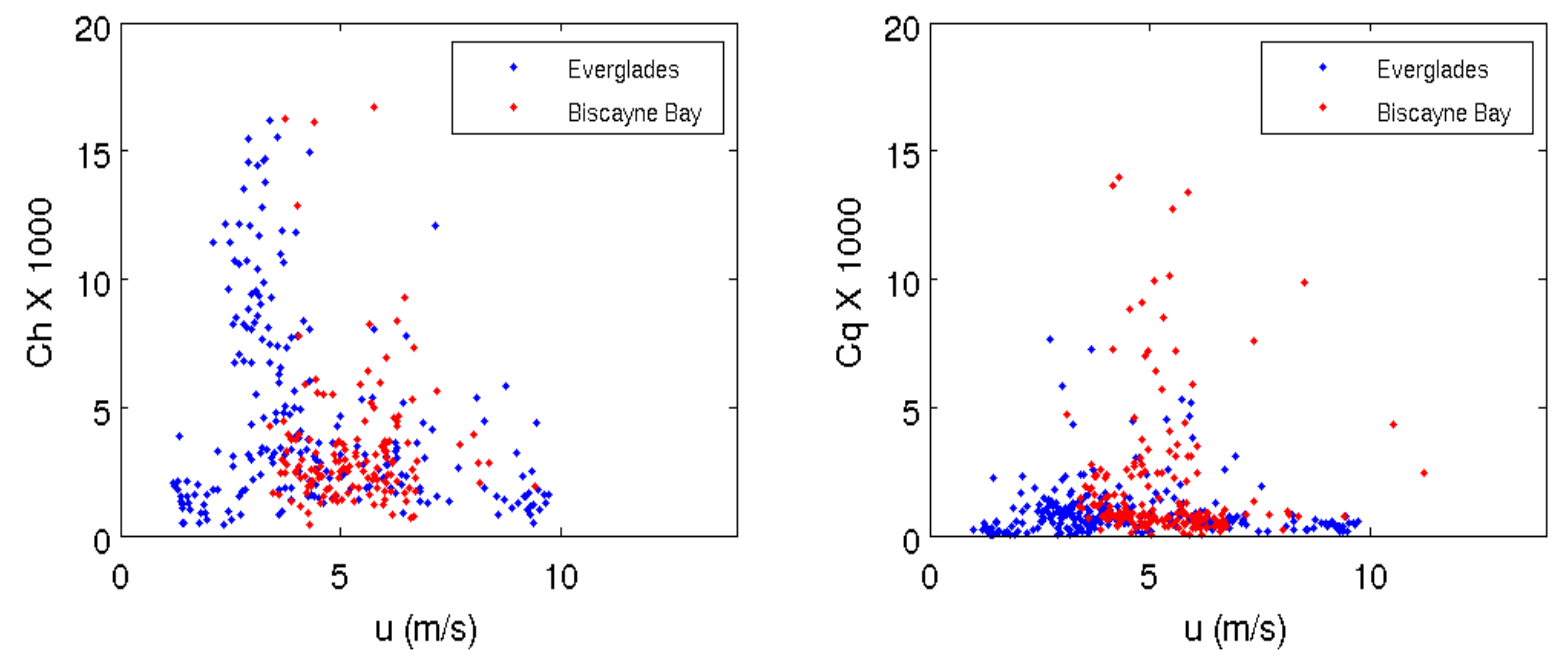

Figure 4.17 Plot of $C_{H}$ (left panel) and $C_{Q}$ (right panel) against 10-m wind speed for both the ENP and BBC sites.

To understand the controlling factors for $C_{H}$ and $C_{Q}$ and the cause for the large spread, $C_{H}$ and $C_{Q}$ are plotted against stability (Figure 4.18). In the left panel, $C_{H}$ 
shows a strong dependence on stability. Large $C_{H}$ values are present in the unstable regime, whereas small $C_{H}$ values are concentrated in the stable regime. It is clear from Figure 4.18 that part of the large spread of $C_{H}$ shown in Figure 4.17 can be attributed to stability. The relatively strong relationship between $C_{H}$ and stability may be attributed to the fact that there is no apparent dependence of $C_{H}$ on wind speed. This lack of dependency contrasts the relationship between $C_{D}$ and stability, which is largely masked by wind speed.

In the right panel of Figure 4.18, there exists no apparent dependence on stability for $C_{Q}$. Instead, large $C_{Q}$ values occur in near neutral conditions. This indicates that a portion of the large spread of $C_{Q}$ may be attributed to the large uncertainty in neutral conditions. Note that a similar phenomenon is also seen for $C_{H}$. As was pointed out before, this is mainly due to the fact that a singularity exists for $C_{H}$ and $C_{Q}$ in neutral conditions. 

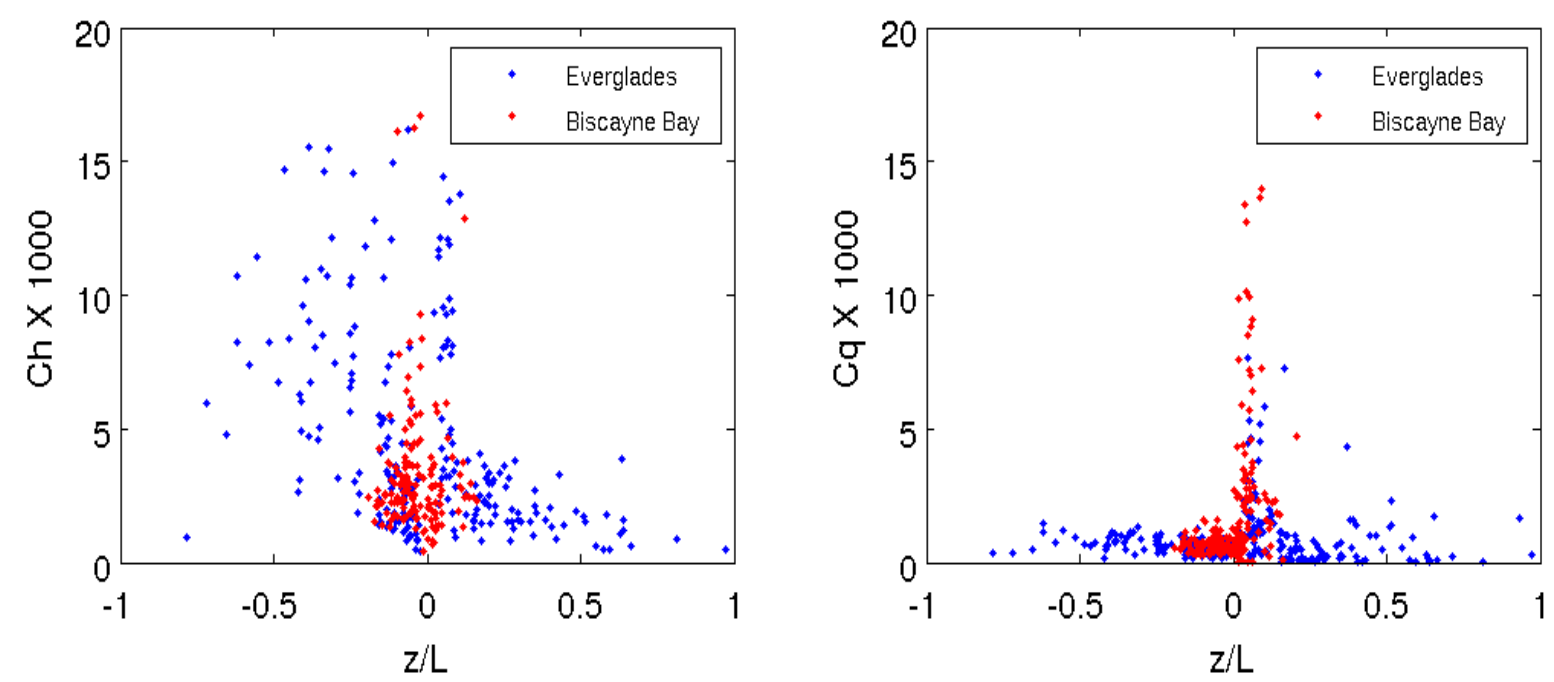

Figure 4.18 Plot of $C_{H}$ (left panel) and $C_{Q}$ (right panel) against $\varsigma=\frac{z}{L}$ for both the ENP and $\mathrm{BBC}$ sites.

Recall in the previous chapter, it was shown that the momentum fluxes are closely tied with turbulent intensity. There is a strong relationship between momentum fluxes and TKE. To see if this is also true for heat and moisture fluxes, Figure 4.19 shows the ratio of $C_{H}$ and $C_{Q}$ to TKE against wind speed plotted in a logarithmic coordinate. Compared to the similar plot of $C_{D}$ (Figure 4.7), the data points in Figure 4.19 are rather scattered, indicating that heat and moisture fluxes are only loosely correlated to turbulent intensity. A consequence of the large spread is that the parameterization of $C_{H}$ and $C_{Q}$ should be treated differently from that of $C_{D}$. 

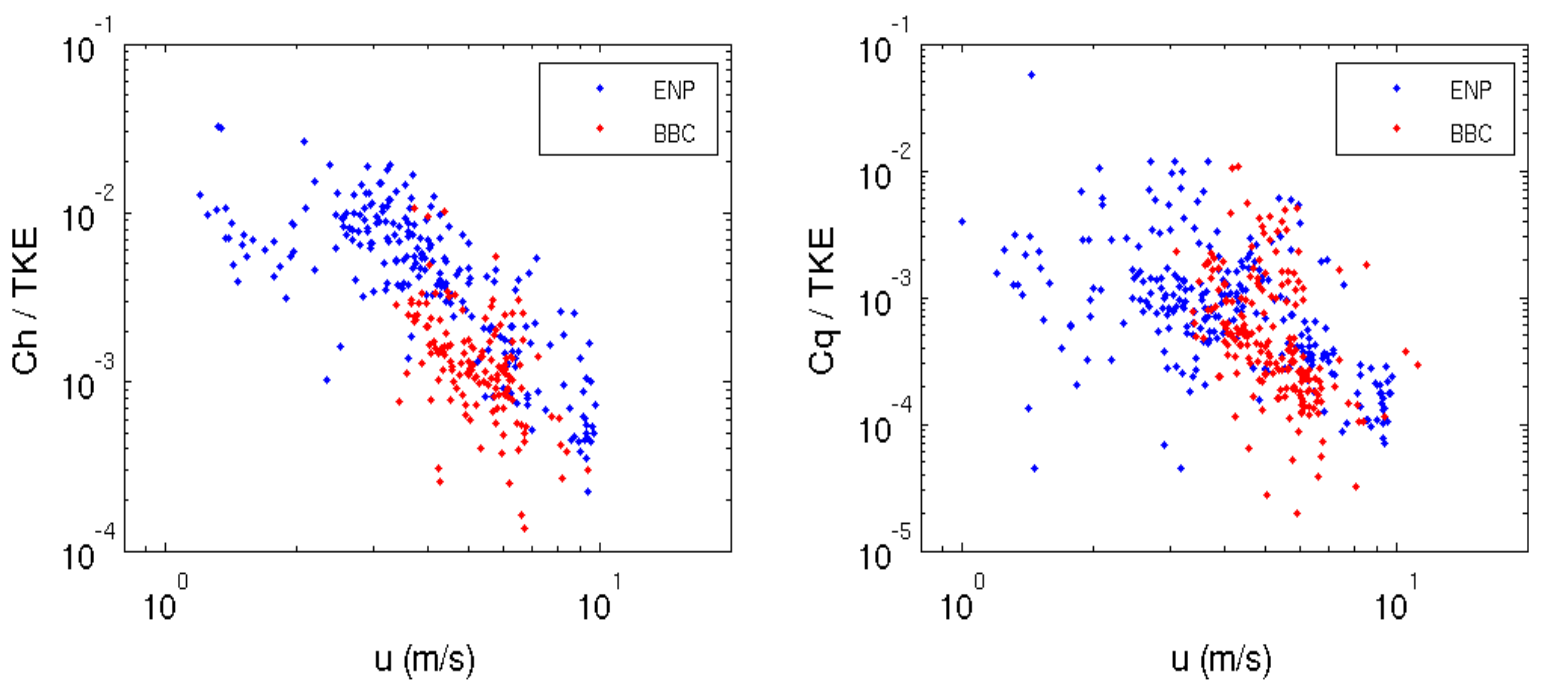

Figure 4.19 Plot (on a logarithmic coordinate) of the ratio of $C_{H}$ to TKE (left panel) and $C_{Q}$ to TKE (right panel) against 10-m wind speed for both the ENP and BBC sites.

To further examine the characteristics of exchange coefficients, Figure 4.20 shows the ratio of $C_{Q}$ to $C_{H}$ and the ratio of enthalpy flux $\left(C_{H}+C_{Q}\right)$ to $C_{D}$ against wind speed. $C_{Q}$ is generally smaller than $C_{H}$. The mean ratio of $\frac{C_{Q}}{C_{H}}$ obtained at the ENP and BBC sites is fairly consistent, with values of 0.23 and 0.29 respectively. One caveat is that the ratio has a large spread. A large difference between the sites is present, as illustrated by the mean ratio of $\frac{C_{Q}+C_{H}}{C_{D}}$. The value obtained at the ENP site $(0.51)$ is more than double that of the BBC site (0.24). However both sites' observations agree that the exchange coefficient for enthalpy flux $\left(C_{Q}+C_{H}\right)$ is substantially smaller than the drag coefficient by a factor of more than two. 


\subsubsection{Tropical Cyclones and Enthalpy Flux}

Using a highly idealized conceptual model, Emanuel $(1985,1989,1995)$ showed there exists a strong sensitivity of the maximum wind speed of tropical cyclones to the ratio of enthalpy flux $\left(\mathrm{C}_{\mathrm{H}}+\mathrm{C}_{\mathrm{Q}}\right)$ to $C_{D}$; in order for a tropical cyclone to attain a max wind speed $>50 \mathrm{~ms}^{-1}$ (Category 3, Saffir-Simpson scale), the ratio of $\frac{C_{Q}+C_{H}}{C_{D}}$ must be $>$ $=0.75$. From the bulk transfer parameterization perspective, our results suggest that a tropical cyclone cannot be enhanced in coastal regions after making landfall even over a saturated surface such as the Everglades since the surface enthalpy flux is simply too weak to support the hurricane's core. This result does not appear to support some previous studies (e.g., Wakimoto and Black 1994; Shen et al. 2002) that suggest landfalling tropical cyclones may temporarily strengthen (or may not be substantially weakened) over a saturated land surface, such as the Everglades. Note that this argument is solely from the bulk transfer model. 

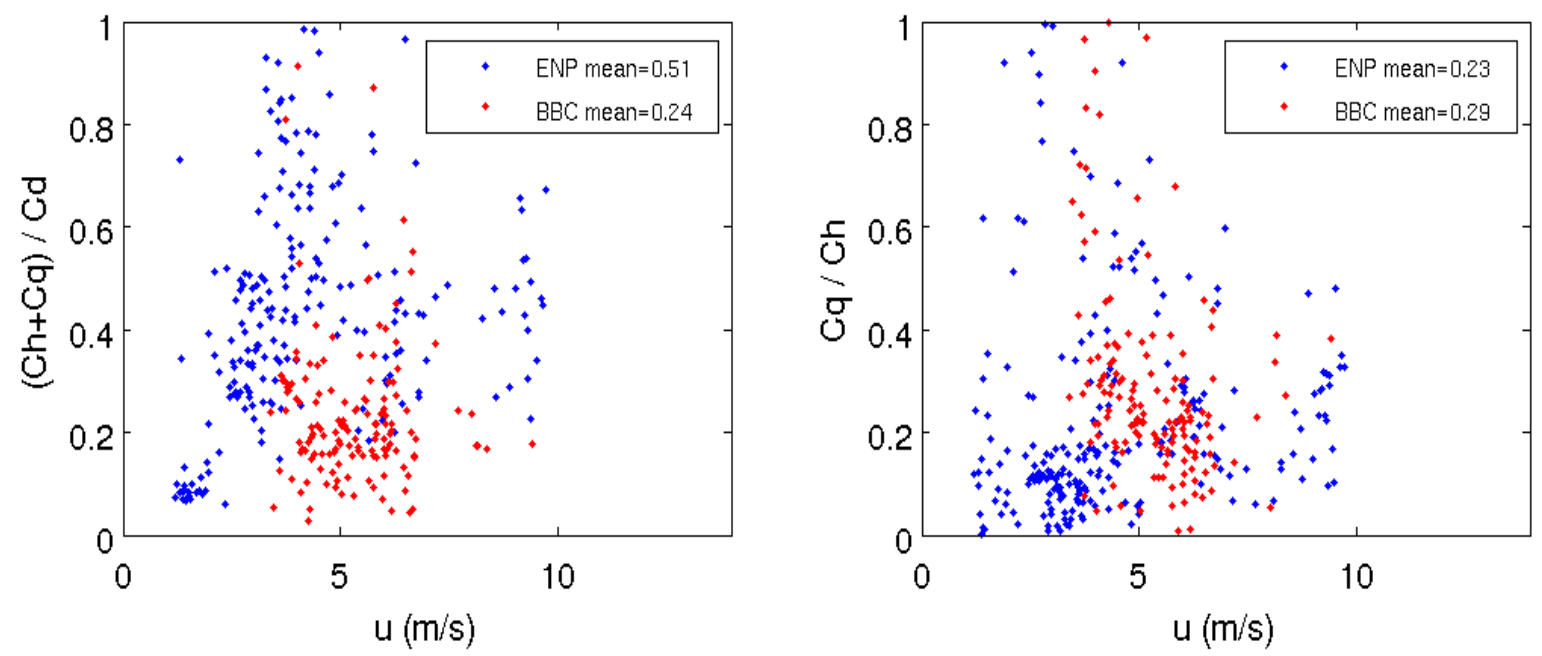

Figure 4.20 Plot of the ratio of enthalpy flux to $C_{D}$ (left panel) and ratio of $C_{Q}$ to $C_{H}$ (right panel) against 10-m wind speed for both the ENP and BBC sites. 


\section{DISCUSSION}

\subsection{Summary}

Vertical turbulent transport in the SL plays a key role in determining the exchange of momentum, heat, and moisture between the atmosphere and the underlying surface. However, in theoretical analyses and numerical simulations, the SL vertical turbulent fluxes are sub-grid scale properties that must be parameterized. A common method to parameterize SL turbulent fluxes is the bulk transfer model in which the turbulent fluxes are represented in terms of reference-level bulk meteorological variables. This is a simple but elegant framework with clear underlying physics. However, the exchange coefficients that relate turbulent fluxes to mean variables cannot be determined by the bulk model itself. They have to be determined empirically by conducting field experiments. In practice, to close the system, the bulk transfer model is often combined with the Monin Obukhov Similarity Theory, which allows the derivation of analytical expressions for exchange coefficients. Although widely used in numerical simulations and data analyses, some characteristics of exchange coefficients predicted by the MOS are not supported by observations. For example, the drag coefficient in neutral conditions predicted by MOS is a constant for a fixed surface roughness. However, observations show that the drag coefficient depends strongly on wind speed at low wind speeds. Moreover, previous studies show that the values of exchange coefficients can change substantially depending on surface conditions, atmospheric stability, wind speed, and other important ambient parameters. Thus, although widely used in meteorology, oceanography, and other geosciences and environmental sciences that involve surface exchange of energy or momentum, an appropriate determination of exchange coefficients remains a challenge. 
This thesis research attempts to investigate the turbulent transport processes in the SL using data collected by the IHRC 10-m PWT at four coastal sites: Everglades, Biscayne Bay, Naples, and Waveland. To extend the analyses, the data previously collected during Hurricane Ivan (2004) are also included in this study. From these sets of high temporal resolution data measured by advanced 3-D wind, temperature, and moisture sensors, turbulent fluxes can be accurately quantified. The objectives of this thesis study are to (1) revisit the issues of drag coefficient in the low wind regime; (2) provide a physically sound explanation for the observed variation of drag coefficient; (3) attempt to extend the classic MOS into the low wind regime; and (4) characterize the exchange coefficients of heat and moisture in coastal regions.

\subsection{Results and Conclusions}

1. This study's analyses show that $C_{D}$ depends strongly on wind speed in the low wind speed regime, which complicates the relationship between $C_{D}$ and atmospheric stability. This explains why the dependence of $C_{D}$ on stability predicted by MOS cannot be shown in our data or in other data published in previous studies. Without considering the effect of wind speed on $C_{D}$, the predicted $C_{D}$ by MOS can be seriously biased, particularly for low wind speeds.

2. It is found that momentum fluxes are well correlated with TKE, indicating that turbulent transport processes are not only controlled by ambient mean properties but are also closely tied with turbulent intensity. The strong relationship between momentum 
fluxes and TKE suggests that the bulk transfer parameterization of momentum fluxes can be derived from analysis of the TKE budget.

3. By further analyzing the TKE budget, a novel but more generalized parameterization framework is proposed for momentum fluxes. With certain assumptions, the new system can be reduced to the classic MOS framework. Most importantly, the new system effectively explains the observed variation of $C_{D}$ in low wind speeds. Moreover, the effect of stability, which has to be determined empirically in the MOS framework, can now be successfully computed from the system itself.

4. Unlike $C_{D}$, the analyses show that $C_{H}$ and $C_{Q}$ do not have an apparent dependence on wind speed. Excluding the influence of wind speed, a clear relationship between $C_{H}$ and stability is shown in this study. However, $C_{Q}$ does not appear to be a function of stability.

5. It is found that $C_{Q}$ is generally smaller than $C_{H}$. The ratio of $C_{Q}$ to $C_{H}$ is about 0.25 . The data also show that the exchange coefficient for enthalpy flux $\left(C_{Q}+C_{H}\right)$ over the Everglades is substantially smaller than (less than half of) the drag coefficient. This result has an important implication: landfalling tropical cyclones may not temporarily intensify or substantially reduce in intensity over a saturated surface such as the Everglades since the surface enthalpy flux is too weak to provide sufficient energy to support a hurricane's core.

In this study, as a result of the limitation of the collected data, we are unable to examine the characteristics of exchange coefficients at high wind speeds. The physics 
underlying the difference among $C_{D}, C_{H}$, and $C_{Q}$ has not been clearly addressed. Investigating these issues will be the focus of a future study. 


\section{REFERENCES}

Al-Jiboori, M. H. Determining of neutral and unstable wind profiles over Baghdad city. Iraqi Journal of Science, 51, 343-350 (2010).

Aubient, M., T. Vesala, and D. Papale, eds. 2013. Eddy Covariance: A Practical Guide to Measurement and Data Analysis. Springer Atmospheric Sciences.

Black, PG, D’Asaro E, Drennan WM, French JR, Niiler PP, Sanford TB, Terrill EJ, Walsh EJ, Zhang JA (2007) Air-sea exchange in hurricanes: Synthesis of observations from the Coupled Boundary Layer Air-Sea Transfer experiment. Bull Amer Meteorol Soc 88:357-374

Busch, N. E.: 1973. 'The Surface Boundary Layer' (Part I), Boundary-Layer Mereorol. 4,213-240.

Businger, J. A., J. C. Wyngaard, Y. Izumi, and E. F. Bradley, (1970): Flux-Profile Relationships in the Atmospheric Surface Layer. Journal of the Atmospheric Sciences, 28, 181-189.

Christen, A., E. Gorsel, and R. Vogt, (2007): Coherent structures in urban roughness sublayer turbulence. RMS International Journal of Climatology, 27, 1955-1968.

Deacon, E. L.: 1968, 'The Leveling Error in Reynolds Stress Measurement', Bull. Amer. Meteorol. Sot. 49, 836

Donelan MA., Haus BK, Reul N, Plant WJ, Stianssnie M, Graber HC, Brown OB, Saltzman ES, (2004) On the limiting aerodynamic roughness of the ocean in very strong winds. Geophys Res Lett 31: L18306

Dyer, A. J. and Hicks, B. B.: 1972, 'The Spatial Variability of Eddy Fluxes in the Constant Flux Layer', Quart. J. Roy. Meteorol. Sot. 98, 206-212.

Emanuel, K. A., 1985: An air-sea interaction theory for tropical cyclones. Part I. $J$. Atmos. Sci.,42, 1062-1071.

- 1989: The finite amplitude nature of tropical cyclogenesis. J. Atmos. Sci.,46, 34313456.

, 1995: Sensitivity of tropical cyclones to surface exchange coefficients and a revised steady-state model incorporating eye dynamics. J. Atmos. Sci.,52, 3969-3976.

Garratt, J. A. (1977): Review of drag coefficients over oceans and continents, Mon. Weather Rev., 105, 915-929. 
Grachev, A. A., Bariteau, L., Fairall, C. W., Hare, J. E., Helming, D., Hueber, J. \& Lang. E. K. Turbulent fluxes and transfer of trace gases from ship-based measurements during TexAQS 2006. J. Geophy. Res., 116, D13110 (2006).

Kaimal, J. C., Wyngaard, J. C., Izumi, Y., and Cote, 0. R.: 1972, 'Spectral Characteristics of Surface Layer Turbulence', Quart. J. R. Meteorol. Sot. 98, 563-589.

Kaimal, J. C., Wyngaard, J. C., Haugen, D. A., Cote, 0. R., Izumi, Y., Caughey, S. J., and Readings, C. J.: 1976, 'Turbulence Structure in the Convective Boundary Layer', J. Atmos. Sci. 33, 2152-2169.

Lanzinger, E., and H. Langmack. Measuring air temperature by using an ultrasonic anemometer. Poster presented at TECO-2005, Bucharest, Romania.

LI-COR Biosciences. (2010). LI-7200 $\mathrm{CO}_{2} / \mathrm{H}_{2} \mathrm{O}$ Analyzer Instruction Manual. Publication No. 984-10564. LI-COR, Inc, Lincoln, NE.

Mahrt L, Vickers D, Sun J, Jensen NO, Jørgensen H, Pardyjak E, Fernando H (2001) Determination of the surface drag coefficient. Boundary-Layer Meteorol 99: 249-276

Mellor, G. L. and T. Yamada, (1974): A hierarchy of turbulent closure models for planetary boundary layers. J. Atmos. Sci., 31, 1791-1806.

Mitsuta, Y. and O. Tsukamoto. (1978): Drag coefficient in light wind. Bull. Disast. Prev. Res. Inst., 28, 25-32.

Monin, A. S., and A. M. Obukhov, (1954): Basic laws of turbulent mixing in the ground layer of the atmosphere. Tr. Akad. Nauk SSSR Geophiz. Inst., 1, 95-115.

Montgomery, R. B., (1948): Vertical Eddy Flux of Heat in the Atmosphere, J. Meteorol. 5, 265-274.

Pond, S.: 1968, 'Some Effects of Buoy Motion on Measurements of Wind Speed and Stress', J. Geophys. Res. 73, 507-512.

Rao, K. G., Narasimha, R. \& Prabhu, A. Estimation of drag coefficient at low wind speeds over the monsoon trough land region during MONTBLEX-90. Geophys Res Lett, 23, 2617-2620 (1996).

Rao, K. Estimation of the exchange coefficient of the heat during low wind convective conditions. Bound-Layer Meteor., 111, 247-273 (2004). 
Schmid H. P., Grimmond C. S. B., Cropley F., Offerle B., Su H. B., (2000):

Measurements of $\mathrm{CO} 2$ and energy fluxes over a mixed hardwood forest in the midwestern United States. Agric For Meteorol., 103, 357-374.

Stull, R. B. 1988. An Introduction to Boundary Layer Meteorology. Kluwer Academic Publishers.

Tanner, C. B. and Thurtell, G. W. : 1969, 'Anemoclinometer Measurements of Reynolds Stress and Heat Transport in the Atmospheric Surface Layer', U.S. Army Electronics Command, R and D Tech. Report, ECOM 66-G22-F, 199 pp.

Wakimoto, R. M., and P. G. Black, 1994: Damage survey of Hurricane Andrew and its relationship to the eyewall. Bull. Amer. Met. Soc., 75, 189-200.

Wilczak, J. M., S. P. Oncley, S. A. Stage, (2001): Sonic Anemometer Tilt Correction Algorithms. Boundary-Layer Meteorology, 99, 127-150.

Zeman, O. (1981): Progress in the modeling of planetary boundary layers. Annual Rev. in Fluid Mech. 13, 253-272. 


\section{APPENDICES}

Appendix A

IHRC Portable Wind Tower

Instrument Specifications 
Table A.1 Instrument specifications for the Gill WindMaster Pro Ultrasonic Anemometer.

Wind Speed

Range:

0 to $65 \mathrm{~m} / \mathrm{s}$ (0 to $145 \mathrm{mph})$

Resolution:

0.01 or $0.001 \mathrm{~m} / \mathrm{s}$

Accuracy $(12 \mathrm{~m} / \mathrm{s})$ :

$<1.5 \% \mathrm{RMS}$

Wind Direction

Range:

0.0 to 359.9 degrees

Resolution:

1 degrees or 0.1 degrees

Accuracy $(12 \mathrm{~m} / \mathrm{s}): \quad 2$ degrees

Speed of Sound

Range:

300 to $370 \mathrm{~m} / \mathrm{s}$

Resolution:

$0.01 \mathrm{~m} / \mathrm{s}$

Accuracy:

$< \pm 0.5 \% @ 20$ degrees C

General

Output sample rate: up to $32 \mathrm{~Hz}$

Sonic temp. range: $\quad-40$ to +70 degrees $C$ 
Table A.2 Instrument specifications for the LiCor LI-7200 $\mathrm{CO}_{2} / \mathrm{H}_{2} \mathrm{O}$ Gas Analyzer System

General

Accuracy: $\quad$ within $2 \%$ of reading

Resolution $\left(\mathrm{H}_{2} \mathrm{O}, \mathrm{CO}_{2}\right)$ : $\quad 0.0067 \mathrm{ppt}$

Operating temp. range: $\quad-25$ to 50 degrees $\mathrm{C}$

Power requirements: $\quad 10.5$ to 30 volts DC

Power consumption: $\quad 12 \mathrm{~W}$ nominal

Path length: $\quad 12.5 \mathrm{~cm}$

Bandwidth: $\quad$ up to $20 \mathrm{~Hz}$

RH range: $\quad$ 0-95\% (non-condensing) 